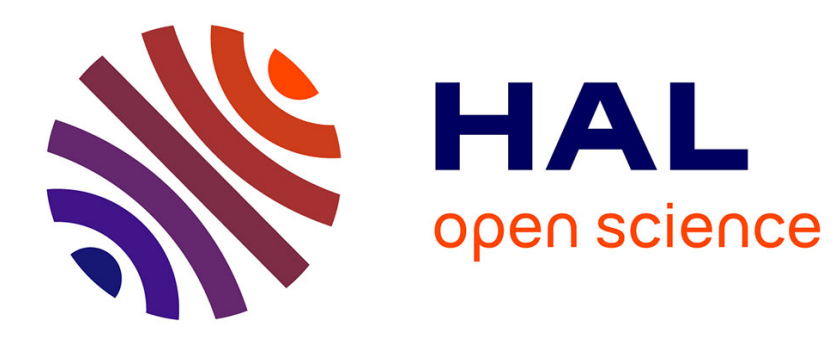

\title{
Formulation of gradient multiaxial fatigue criteria
}

Duy-Hao Luu, Habibou Maitournam, Q. S. Nguyen

\section{To cite this version:}

Duy-Hao Luu, Habibou Maitournam, Q. S. Nguyen. Formulation of gradient multiaxial fatigue criteria. International Journal of Fatigue, 2014, 61, pp.170-183. 10.1016/j.ijfatigue.2013.11.014 hal01216167

\section{HAL Id: hal-01216167 \\ https://hal.science/hal-01216167}

Submitted on 15 Oct 2015

HAL is a multi-disciplinary open access archive for the deposit and dissemination of scientific research documents, whether they are published or not. The documents may come from teaching and research institutions in France or abroad, or from public or private research centers.
L'archive ouverte pluridisciplinaire HAL, est destinée au dépôt et à la diffusion de documents scientifiques de niveau recherche, publiés ou non, émanant des établissements d'enseignement et de recherche français ou étrangers, des laboratoires publics ou privés. 


\title{
Formulation of gradient multiaxial fatigue criteria
}

\author{
D.H. Luu ${ }^{\mathrm{a}, \mathrm{b}}$, M.H. Maitournam ${ }^{\mathrm{a}}$, Q.S. Nguyen ${ }^{\mathrm{a}}$ \\ ${ }^{a}$ Laboratoire de Mécanique des Solides (LMS), CNRS UMR 7649 \\ Ecole Polytechnique - 91128 Palaiseau Cedex, France. \\ ${ }^{\mathrm{b}}$ Université des Sciences et des Technologies de Hanö̈ (USTH), Hanö̈, Vietnam. \\ Correspondence: D.H. Luu; E-mail: luuduyhao@gmail.com \\ M.H. Maitournam; E-mail: maitournam@lms.polytechnique.fr \\ Q.S. Nguyen; E-mail: son@lms.polytechnique.fr
}

\begin{abstract}
A formulation of gradient fatigue criteria is proposed in the context of multiaxial high-cycle fatigue (HCF) of metallic materials. The notable dependence of fatigue limit on some common factors not taken into account in classical fatigue criteria, is analyzed and modeled. Three interconnected factors, the size, stress gradient and loading effects, are here investigated. A new class of fatigue criteria extended from classical ones with stress gradient terms introduced not only in the normal stress but also in the shear stress components, is formulated. Such a formulation allows to capture both "size" and gradient effects, as well as to cover a wide range of loading mode, then can model both phenomena "Smaller is Stronger" and "Higher Gradient is Stronger". Gradient versions of some classical fatigue criteria such as Crossland and Dang Van are provided as illustrations.
\end{abstract}

Key words: gradient multiaxial fatigue; size effect; gradient effect; loading effect; High Cycle Fatigue

\section{Introduction}

In recent years there has been an increasing interest in developing fatigue criteria for metals capable of dealing with high stress gradient (around notches, voids, con- 
tacts, etc.) and particular issues related to small scales. Examples are found, on the one hand in notches and fretting problems $[1 ; 2 ; 3 ; 4 ; 5 ; 6]$, and on the other hand in problems related to small electronic components and electro-mechanical devices. At sufficiently small sizes, some factors (size, gradient and loading effects) which effects on fatigue limits are inherently not captured by classical fatigue criteria, become important and must be taken into account through new criteria. Among them, experimental evidences show three interconnected ones: size effect, gradient effect $^{1}$ and loading effect ( $\left.c f .[7 ; 8 ; 9 ; 10 ; 11 ; 12]\right)$. A visible general correlation between these factors is that, "the smaller the size, the higher the gradient, then the higher fatigue resistance". There are also cases where the gradient exists but independent from the size, although both influence on material strength (e.g. residual surface stress cases). For the sake of further analyses, it requires to clarify what are the sources of the size effect by isolating it from the gradient effect. Size effect is commonly considered as the pure size effect related to the metallurgical defects and heterogeneity of material, and is proved insignificant compared to the other at the considered scale (e.g. tension-compression fatigue test in Fig. 5, [13; 7]). Then a preliminary qualitative remark is that, such a pure size effect just is a part, but not enough to explain the fact well known as "Smaller is Stronger" that we observe in fatigue tests.

The gradient effect is another factor which may help to interpret that fact. Such effect, termed here "Higher Gradient is Stronger", is roughly related to three sources: boundary condition, loading mode and size. The first is associated with constraints on dislocation glide (passivated surfaces and interfaces, boundary layers, etc.); the second concerns loading type which decides the spatial stress distribution state in the solid (null gradient in tension-compression, non-zero gradient in bending, etc.); the last is associated with the size (e.g. geometry and grain sizes). For instance, in bending test, the smaller the beam radius the higher the stress gradient (and the higher the fatigue limit). Experimental results $[14 ; 7]$ on the variation in fatigue strength at various radii conclude to the dominance of the gradient effect upon the pure size effect. Then the sources of the gradient effect prove two things: first, "Smaller is Stronger" experimentally observed is mainly attributed to the gradient

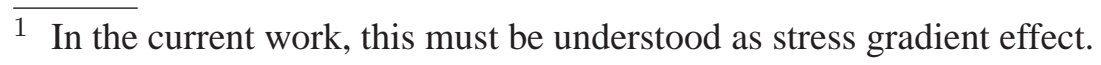


effect in the cases considered here, rather than totally to the pure size effect as usually believed; second, the gradient effect, i.e. "Higher Gradient is Stronger", is really a phenomenon different from the size effect.

All previous analyses for both the size and gradient effects imply that although the size and gradient effects are intimately interconnected and usually confused in the literature, they are actually two distinct phenomena. The former only contributing in part to "Smaller is Stronger" and requiring to be modeled by other approach, is negligible compared to the latter and thus left out in the current study; whereas the latter is not only "Higher Gradient is Stronger" but also a main factor contributing to "Smaller is Stronger" that we observe, and is the object of study here. In brief, from phenomenological aspect, "Higher Gradient is Stronger" is naturally related to the gradient effect only, while "Smaller is Stronger" is related to both pure size and gradient effects where the latter is dominant. Then "Smaller is Stronger" here is just a "visible image" of gradient effect rather than the size effect from mechanical point of view. From phenomenological point of view, "Smaller is Stronger" is however an experimentally observed fact that evokes an intuitive relation to the size rather than the gradient. For this reason, henceforth in this research, the terminology "size effect" (placed within quotes) is still used for "Smaller is Stronger", but as an apparent size effect; and the terminology gradient effect is used for "Higher Gradient is Stronger". In such a sense, an important conclusion drawn is that, taking into account only gradient effect (related to all its sources) is enough to capture both "size effect" and gradient effect on fatigue resistance.

In this study, only cases where the gradient effect is present apart from the inherent pure size effect, are considered. As in [7], the notch effect - regarded as a particular case of the gradient effect, is left out in the study restricted to macroscopically elastic behavior or stabilized elastic shakedown state [15]. In such a context and along with the notable conclusion above, Gradient Fatigue Criteria with stress gradient terms introduced are capable to capture the "size", gradient and loading effects, and thus to model both phenomena "Smaller is Stronger" and "Higher Gradient is Stronger", as found in the applications considered here.

Classical fatigue criteria without material length scale predict no size, gradient neither loading effects. The objective is to establish a new class of fatigue criteria for 
considering the previous factors. Existing approaches dealing with such problems are $(c f .[8 ; 9 ; 10 ; 11 ; 12]):(i)$ critical layer of Flavenot and Skally [16]; (ii) distance approaches such as: effective distance approach of Pluvinage [4], Qylafku et al. [5]; theory of critical distances, Taylor [2], Araujo et al. [3]; (iii) nonlocal approaches such as: maximum stressed-strained volume by Sonsino et al. [17]; energy based criterion of Palin-Luc and Lasserre [18]; volumetric energy based criterion of Banvillet et al. [9] and Palin-Luc [10]; gradient method proposed by Brand and Sutterlin [19; 20]; (iv) local approaches such as: gradient dependent criterion of Papadopoulos and Panoskaltsis [7]; that of Ngargueudedjim et al. [21], and several derivatives based on this work [7] proposed by Fouvry et al. [1; 22] and Weber [12] (gradient version of the criterion of Robert [23], and that of Fogue [24; 25]), etc.

The review of Papadopoulos and Panoskaltsis [7] is re-used and developed to make more clear the connection as well as the distinction between the effects by analyzing the role of each dimension of specimen in fatigue resistance. It is shown that two issues remain: first, the non-effect of the shear stress gradient on fatigue limits is only found for some metals - but not all; second, the influence of the stress gradient amplitude must be clarified. Thereby, in the spirit of [7], gradient fatigue criteria extended from classical ones with stress gradient terms are proposed and validated to clarify the issues. The main idea is to maintain the general framework of the classical fatigue criteria, but to embed into it gradient terms which enable to describe the effects concerning the stress heterogeneous distribution. Three steps are done: first, the dependence of fatigue limit on the previous factors in the cases of uniaxial stress cyclic loadings is phenomenologically analyzed; second, the stress gradient fatigue criteria which capture the previous factors are established; and finally, a generalization to multiaxial loadings is performed and some applications are provided.

The outline of the work is as follows. Section (2) focuses on re-analyzing existing experiments on gradient, size and loading effects; in Section (3), basing on these analyses as well as notable observations and using as a basis classical fatigue criteria in the spirit of [7], new criteria with stress gradient terms entering not only in the normal stress but as well in the shear stress parts, are proposed in the context of macroscopic elasticity. Such a formulation allows the new criteria to capture the 
phenomena $^{2}$ only by means of gradient terms. These criteria are generalized under multiaxial loadings to be a new class of stress gradient multiaxial fatigue criteria; in Section (4) and (5), some classical fatigue criteria such as Crossland and Dang Van are extended within such framework; Section (6) is devoted to their numerical implementation; and finally, Sections (7) and (8) are discussions and conclusions.

\section{Analyses of gradient fatigue tests: size, gradient and loading effects}

In this section, analyses on single component zero and non-zero gradient fatigue tests from the literature, including two groups, uniaxial normal stress and shear stress tests, are made to clarify the size, stress gradient and loading effects on fatigue limits. The tests exempt from the size and gradient effects, are used as reference. A special attention is also paid on the interpretation of the three effects and their relation as well as the capacity of either eliminating or integrating them into "gradient terms" for some cases. Analyses and preliminary conclusions drawn here for single component fatigue tests are generalized to formulate new gradient fatigue criteria under multiaxial cyclic loadings.

\subsection{Uniaxial normal stress cyclic loading}

\section{a) Experimental observations and interpretation of stress gradient effect}

Some analyses of [7] and [12] are reported here on fatigue endurance of metals in bending or tension-compression tests. Two respective distinct groups of results, uniaxial normal cyclic stress states with non-zero and zero normal stress gradients, respectively, allow to draw some comments about the normal stress gradient effect and about the possibility of integrating the loading effect into gradient effect. In the first example, a well-established experimental fact is always found: for the same smooth geometry and material, and the same nominal stress $\sigma_{\max }$ (Fig. 1(a)), the specimen in fully reversed tension-compression test sustains lower nominal fatigue

$\overline{2}$ In this study, these effects are captured in the sense that the gradient effect has to be present as prerequisite - to which the loading effect is naturally attached, whereas and the pure size effect is proved unimportant compared to the others. 


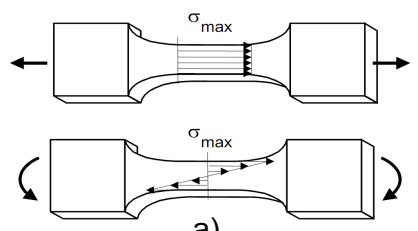

a)

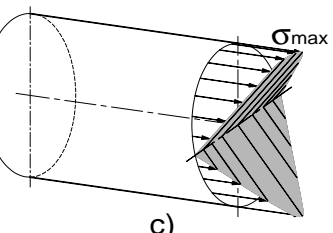

c)

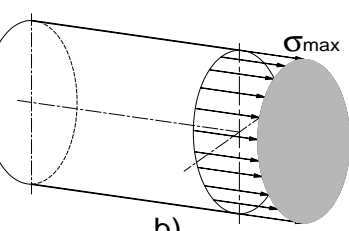

b)

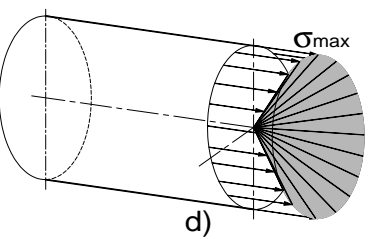

Fig. 1. Stress distribution types in fatigue tests of the same specimen: (a) tension-compression vs. bending tests; (b-c-d) tension-compression vs. rotative bending vs. plane bending (cf. Weber [12])

stress than in fully reversed bending test. Or similarly but in another observation $[35 ; 12 ; 7]$ : a large number of experiments proved that the fully reversed bending fatigue limit $\mathrm{f}_{-1}$ (rotative bending, or plane bending) is always higher than the homologue $\sigma_{-1}$ in fully reversed tension-compression test for smooth samples with the same geometry and material (Tab. 1). This experimental fact is attributed to the "beneficial gradient effect" [7], which exists in bending but not in tension. The

\begin{tabular}{|l|c|c|c|c|}
\hline \multicolumn{1}{|c|}{ Materials } & $\begin{array}{c}\mathrm{N}_{\mathrm{D}} \\
(\text { cycles })\end{array}$ & $\begin{array}{c}\sigma_{-1} \\
(\mathrm{MPa})\end{array}$ & $\begin{array}{c}\mathrm{f}_{-1} \\
\text { (rotative bending) } \\
(\mathrm{MPa})\end{array}$ & $\begin{array}{c}\text { Difference between } \\
\sigma_{-1} \text { and } \mathrm{f}_{-1} \\
(\%)\end{array}$ \\
\hline Steel 30NCD16 & $10^{6}$ & 560 & 658 & +17.5 \\
\hline Steel XC18 & $10^{6}$ & 273 & 310 & +13.6 \\
\hline Iron cast GS61 & $10^{6}$ & 245 & 280 & +14.3 \\
\hline Steel 35CD4 & $10^{7}$ & 558 & 581 & +4.1 \\
\hline
\end{tabular}

Table 1

Comparison between the fully reversed tension-compression and rotative bending fatigue limits of smooth specimens with the same geometry and material, for different materials (Results of Palin-Luc [35], synthesized by Weber [12])

second experimental example illustrates and makes more clear the point of view "beneficial gradient effect" and also roughly deals with the size effect discussed more in detail in the next analyses. Fig. 2 presents the experimental results obtained on smooth circular tubes subjected to tension-compression or rotating bend- 
ing. In tension-compression the stress gradient is zero, the results exhibit a slight increase tendency in fatigue limit when the radius of test specimens decreases. Because of the absence of stress gradient, this variation of the fatigue limit may be considered as a pure size effect analyzed later. With the counterparts in rotative bending, however, a strong increase tendency in fatigue limit with decreasing radius and an asymptotic value when the radius increases, are found. Apart from the pure size effect as in the tension-compression case, this strong increase tendency of fatigue limit with the small radius as well as the saturation or insensitivity tendency with the large enough radius again, can be only attributed to the beneficial gradient effect which increases as the radius decreases and vice versa. The two examples

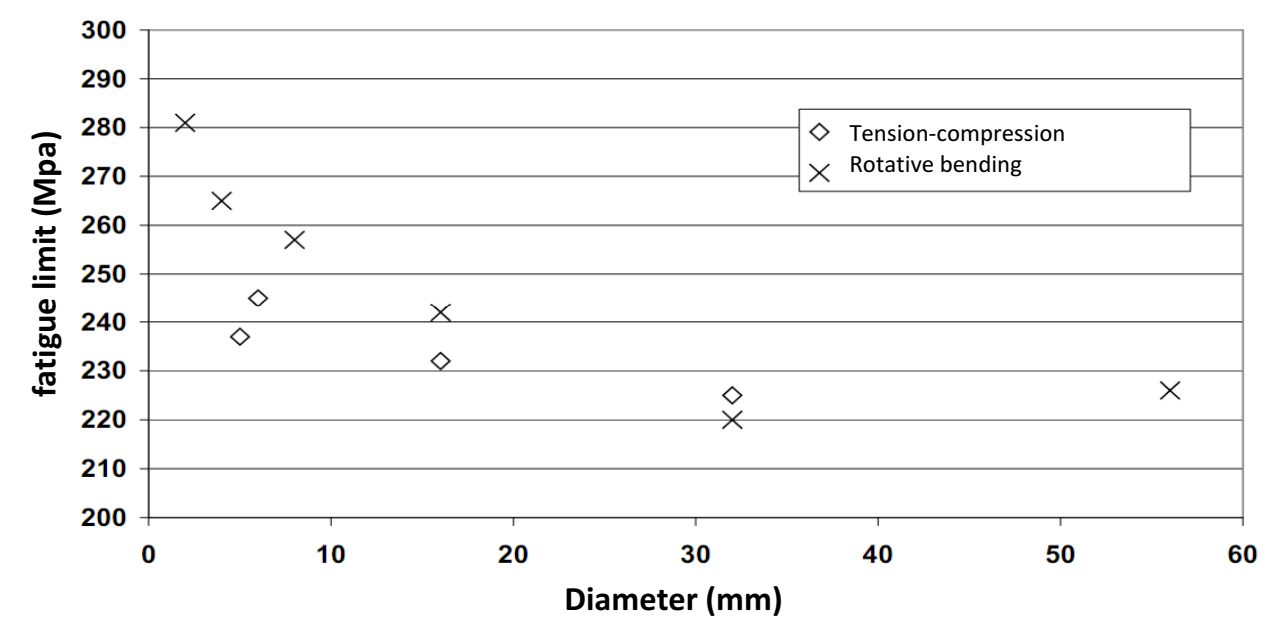

Fig. 2. Evolution of the fully reversed tension-compression and rotative bending fatigue limits of smooth specimens with the same geometry and material according to their radii (Results of Massonnet [27], synthesized by Weber [12])

above only sketched the influence of the pure size and gradient effects on fatigue limits. Besides these two factors, it remains the loading effect within the context of the current treatment. The study of the loading effect needs to be now put into the consistent framework with the previous others, to thoroughly examine all of three, from probabilistic point of view of fatigue damage related to metallurgical defects. Indeed, the difference in fatigue limit in the various test cases of the above examples can be explained from a statistical point of view: the larger these volumes are, the larger the number of defects, i.e. the more the probability of fatigue damage of the specimen is. 
First, consider the pure size effect through constant moment tests on samples of the same material, bending moment and radius - i.e. the same nominal maximum stress $\sigma$ and stress gradient, but different lengths (data of [14], represented by [7]). As shown in Fig. 4(a), the bending fatigue limit always increases with the decrease in the specimen length. For the same radius, the volume of the most loaded zone decreases with the decrease in the length. Hence, a conclusion drawn about the "pure size effect" is: for the same instant stress distribution as well as nominal maximum stress and material, the smaller the sample size is, the smaller the volume of the most stressed zone is, the higher the fatigue limit is.

Second, three types of tests, in tension-compression, rotative bending and plane bending, for the smooth specimens with the same geometry and material, subjected to the same nominal maximum stress $\sigma_{\max }$, are now examined (Fig. 1(b-c-d)) in order to make clear the stress gradient effect. The fatigue limits [27] are respectively decreasing as as reported in Fig. 2 and Tab. 2, [12]. For materials with defects, this phenomenon can be explained from a probabilistic point of view. In fact, the common feature of the three tests is, the critical points on their cross-section are subjected to the same stress state. However, the volumes of the most loaded zones are different. In descending volume order, they are tension-compression, rotative bending and plane bending, corresponding to increasing order of fatigue limits. The stress gradient leads to a disparity of the stress distribution, and with the same nominal maximum stress, that also leads to the diminution of the volumes of the most stressed zones, i.e. to the raise of fatigue resistance. The stress gradient is then a quantity able to represent and model all those informations, notably fatigue resistance. Another explanation is related to the average stress in a representative volume element (RVE) [6], which is different between the three tests for the critical point, during a fatigue cycle. This stress is equal to the maximum stress for the tension-compression tests, whereas it is reduced by the presence of a gradient for the bending tests. Therefore, the maximum stress in the RVE and the stress gradient are two relevant quantities for the fatigue resistance; they will be used in the formulation of fatigue criteria taking these phenomena into account. 
Third, the loading effect implies the influence of loading mode on fatigue limit. For instance, for the same geometry, material and nominal maximum stress, plane bending and rotative bending give different fatigue limits. In fact, the rotative bending induces a more important circumferential stress gradient due to rotation. The loading effect of the rotative bending, as just explained, can be captured by using probabilistic approach or possibly by averaging stresses on a relevant RVE.

To summarize, the pure size, stress gradient and loading mode are three factors influencing on fatigue. Their close connection can be interpreted either under the probabilistic failure aspect as just discussed, or under the average stress in the RVE, although their manifestations are not totally identical. In this study, gradient approaches will be developed to represent some of these phenomena.

\begin{tabular}{|l|c|c|c|c|}
\hline \multicolumn{1}{|c|}{ Materials } & $\begin{array}{c}\mathrm{N}_{\mathrm{D}} \\
\text { (cycles) }\end{array}$ & $\begin{array}{c}\mathrm{f}_{-1}^{\text {rotative }} \\
\text { (rotative bending) } \\
(\mathrm{MPa})\end{array}$ & $\begin{array}{c}\mathrm{f}_{-1}^{\text {plane }} \\
\text { (plane bending) } \\
(\mathrm{MPa})\end{array}$ & $\begin{array}{c}\text { Difference between } \\
\mathrm{f}_{-1}^{\text {rotative }} \text { and } \mathrm{f}_{-1} \text { plane } \\
(\%)\end{array}$ \\
\hline Steel 30NCD16 & $10^{6}$ & 658 & 690 & +4.9 \\
\hline Steel XC18 & $10^{6}$ & 310 & 332 & +7.1 \\
\hline Iron cast GS61 & $10^{6}$ & 280 & 294 & +5.0 \\
\hline Steel 35CD4 & $10^{7}$ & 581 & 620 & +6.7 \\
\hline
\end{tabular}

Table 2

Comparison between the fully reversed rotative bending and plane bending fatigue limits for different metals of smooth specimens of the same geometry and material (Results of Palin-Luc [35], synthesized by Weber [12])

\section{b) Typical fatigue tests}

The differences between four-point bending tests and cantilever bending experiments allow to point out the distinction between pure size and gradient effects. In the former, the bending moment is the same at any time in the interval $L \leq x \leq L+l$ and equal to $M=F L$ (Fig. 3(a)). The bending stress $\boldsymbol{\sigma}$ and its gradient $\boldsymbol{Y}$ for $L \leq x \leq L+l$ and $-R \leq y \leq R$ are then:

$$
\begin{aligned}
& \boldsymbol{\sigma}=\sigma_{x x} e_{x} \otimes e_{x}, \quad \sigma_{x x}=\frac{F L}{I} y \\
& \boldsymbol{Y}=\nabla \boldsymbol{\sigma} \text { with } \sigma_{x x, x}=0, \quad \sigma_{x x, y}=\frac{F L}{I}=\frac{\sigma_{x x}}{R}, \sigma_{x x, z}=0
\end{aligned}
$$




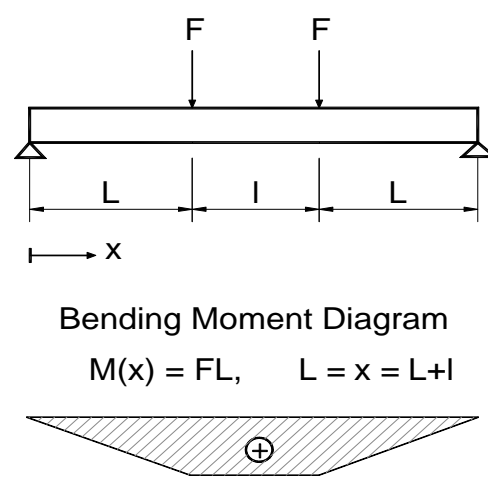

a)

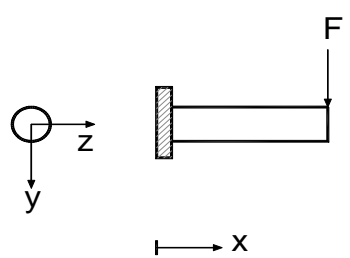

Bending Moment Diagram

$M(x)=-F(L-x)$

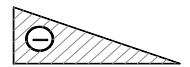

b)

Fig. 3. Four-point bending (constant moment) and cantilever bending tests: (a) four-point bending; (b) cantilever bending [7].

in which Eq. (2) is written for the most stressed points, i.e. points located at $L \leq$ $x \leq L+l$ and at $y= \pm R$. In both Eqs. (1) and (2), all components not mentioned are null. The notations $\sigma_{x x, x}, \sigma_{x x, y}$ and $\sigma_{x x, z}$ mean partial derivative of $\sigma_{x x}$ relative to respectively $x, y$ and $z$.

In the cantilever bending test the bending moment is: $M=-F(L-x)$ (Fig. 3(b)). The bending stress and its gradient for $0 \leq x \leq L$ and $-R \leq y \leq R$ are given by:

$$
\begin{aligned}
& \boldsymbol{\sigma}=\sigma_{x x} e_{x} \otimes e_{x}, \quad \sigma_{x x}=\frac{-F(L-x)}{I} y \\
& \boldsymbol{Y}=\nabla \boldsymbol{\sigma} ; \quad \sigma_{x x, x}=\frac{F}{I} y=\frac{-\sigma_{x x}}{L}, \sigma_{x x, y}=\frac{-F(L-x)}{I}=\frac{\sigma_{x x}}{R}, \sigma_{x x, z}=0
\end{aligned}
$$

Eq. (4) is written for the critical points, i.e. those at $x=0$ and $y= \pm R$.

In their work, [7] did distinguish clearly the pure size and gradient effects on fatigue limits, and both obviously concern the specimen size in diverse manners. Now it is worthy recalling and making more clear the role each specimen size (such as the length $L$ and radius $R$ of the beam) plays in the pure size and gradient effects on fatigue strength. The aim of such analysis is to answer to a question: "Is it possible to formulate fatigue criteria which can include these both effects in a certain sense just by introducing in classical criteria appropriate "gradient terms"? As wellknown, according to many authors the pure size effect should be addressed within the context of statistical approaches. To answer to the question, the role of each sample dimension must be clarified. First of all, it is confirmed that both length and radius of specimens affect on the fatigue limit (Fig. 4): the larger the radius and/or the length, the lower the fatigue endurance. But a more important question is: by 
means of which effect they influence on the fatigue resistance (through the pure size effect, or the gradient effect, or even both simultaneously)? On the one hand, the influence of $L$ on the fatigue limit is a well-established experimental fact after the results of [14] synthesized by [7] in Fig. 4(a). On the other hand, in view of Eq. (2) showing the independence of the normal stress gradient on $L$, thus the role of $L$ in the fatigue limit in four-point bending is clearly realized through solely the pure size effect not gradient effect.
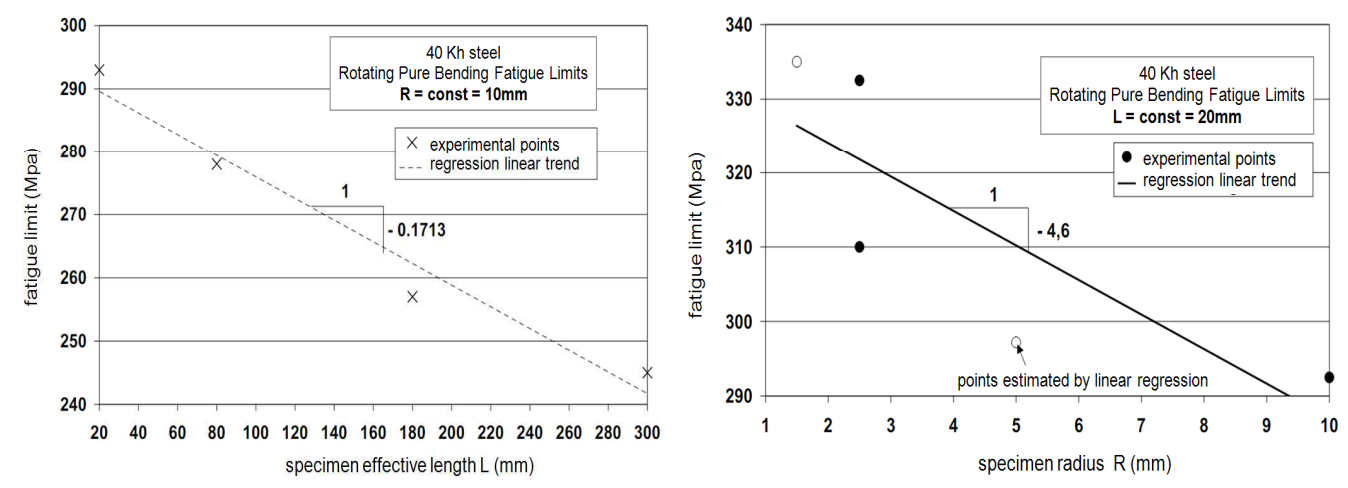

Fig. 4. Constant moment bending fatigue limit data: (a) constant radius $R$; (b) constant length L (Results of Pogoretskii and Karpenko [14], represented by Weber [12])

On the contrary, apart from the pure size effect, the gradient effect is present as the normal stress gradient is not zero and is also $R$ dependent (Eq. 2).

The quantitative estimate of the contribution of the pure size effect made in [7], using the results of the constant moment tests on specimens of the same radius but different lengths, is recalled and used. The slope of the linear trend observed for the (fatigue limit- $R$ ) data in Fig. 4(a) is much higher than the one for the (fatigue limit$L)$ data in Fig. 4(b). This shows that the gradient effect is an order of magnitude higher than the pure size effect. It eventually results in, for the case of constant moment tests, a preliminary conclusion that, an appropriate introduction of the normal stress gradient terms in the expression of fatigue criteria is enough to reproduce the experimental results.

The influence of $L$ and $R$ on the fatigue limit are now realized by means of the inherent pure size effect and the gradient effect as both the length $L$ and the radius $R$ are present in the expression of the normal stress gradient (Eq. 4). From the previ- 
ous observations, one can conclude that a presence of normal stress gradient terms in the formula of fatigue criteria, such as Eq. (4), is enough to accurately model these fatigue tests.

Besides this analysis, the experiments of [13], performed under fully reversed tensioncompression on specimens of various sizes, manifested a negligibly small pure size effect on the observed fatigue limits. These experimental data are depicted in Fig. 5 for cylindrical specimens of a mild steel and a nickel-chromium steel, where the observed fatigue limits are plotted against the specimen radii. It seems that no systematic pure size effect related to $R$ exists. In another class of results, Fig. 2 indicates a slight increase tendency of tension-compression fatigue limit with the decrease in specimen radius. A conclusion drawn from these results is, the pure size effect is neolioihle at least within the size ranoe under consideration

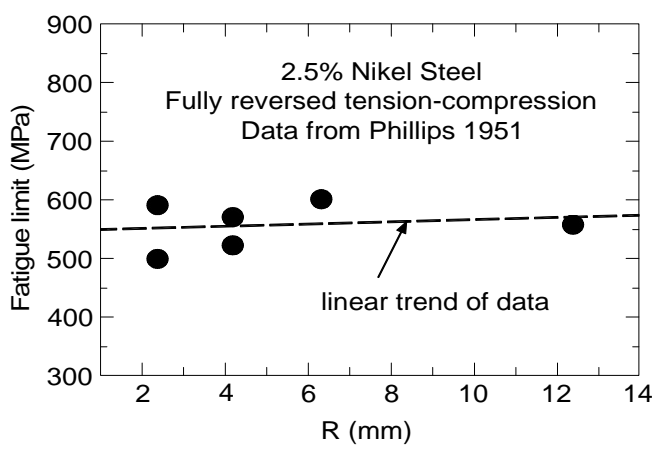

a)

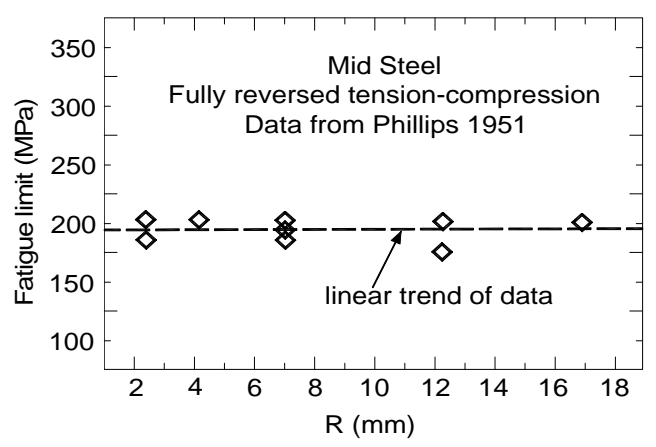

b)

Fig. 5. Fully reversed tension-compression fatigue limit data (Results of Phillips and Heywood [13], represented by Papadopoulos and Panoskaltsis [7])

\subsection{Shear stress cyclic loading}

Cyclic torsion tests (fully reversed and/or asymmetrical torsion tests) from the literature are examined in this section. Torsion tests intrinsically exhibit shear stress gradients, which are therefore always present in the cases considered here. The comparison of the torsion fatigue limit between different superimposed mean torque tests, i.e. different mean shear stresses as well as its gradients for the same smooth geometry and material, is re-analyzed.

The experimental result, clearly demonstrated by the compilation in [36], is that the 
fatigue limit in torsion is the same in fully reversed and in any asymmetrical torsion tests for the same smooth geometry and material. Basing on this fact, [7] did conclude the independence of the fatigue limit from the shear stress gradient effect for some metals. In view of this, [7] did not introduce any gradient term concerning shear stresses in their fatigue criteria. Departing from this result, we add the argument that such an independence of the fatigue limit does not ensure a similar independence from the amplitude of the shear stress gradient. Thus, the amplitude of the shear stress gradient is introduced in the relevant component of fatigue criteria (sec. 4.3).

To consider this capability, the shear stress state and its gradient in torsion tests for $-R \leq r \leq R$, are written down:

$$
\begin{aligned}
& \boldsymbol{\sigma}=\sigma_{x z}\left(e_{x} \otimes e_{z}+e_{z} \otimes e_{x}\right) ; \quad \sigma_{x z}=\frac{M}{I} r=\frac{M}{I} \sqrt{x^{2}+y^{2}} \\
& \boldsymbol{Y}=\nabla \boldsymbol{\sigma} ; \quad\left[\sigma_{x z, x}=\frac{M}{I} \frac{x}{\sqrt{x^{2}+y^{2}}}=\sigma_{x z} \frac{x}{R^{2}}, \sigma_{x z, y}=\frac{M}{I} \frac{y}{\sqrt{x^{2}+y^{2}}}=\sigma_{x z} \frac{y}{R^{2}}, \sigma_{x z, z}=0\right]
\end{aligned}
$$

where Eq. (6) is written for the maximum strained points, i.e. $r=R$.

The influence of $R$ on the fatigue limit, experimentally observed as in Fig. 6 after [27] is concretized through the pure size effect and the shear stress gradient amplitude effect presumably. According to the previous analyses, the pure size effect concerning $R$ is regarded as negligible compared to the latter. Therefore the introduction of a shear stress gradient amplitude term is sufficient to reproduce the experimental results.

\subsection{Discussion}

Analyses in the section 2.1 show that: (i) the gradient effect on four-point bending fatigue limits related to the length is null whereas the pure size effect related to the length is negligible compared to both pure size and gradient effects related to the radius. (ii) the gradient effect on tension-compression fatigue limits related to all dimensions is null whereas the pure size effect related to the radius can also be negligible, at least within the radius size range under consideration. Analyses in the section 2.2 prove that: (iii) for the considered metallic materials, the shear stress gradient effect on torsion fatigue limits through all dimensions is null and the role of the stress gradient amplitude effect is possible. 
These estimations allow to preliminarily confirm the possibility to formulate new gradient fatigue criteria well reproducing the analyzed experimental results. In brief, the above indepth comparative analysis demonstrates the negligibility of the pure size effect, whereas affirms the strong influence of the normal stress gradient as well as the non-influence of the shear stress gradient, and especially allows supposing the possible role of the shear stress gradient amplitude. Indeed, a dependence of the pure torsion fatigue limit of a cylinder on its radius is only attributed to the shear stress gradient amplitude effect as both normal and shear stress gradient effects are here null while the pure size effect is always insignificant. Hence, apart from a gradient term introduced into the normal stress component as proposed in [7], another term of gradient amplitude into the shear stress component of any fatigue criterion is indispensable (most visibly for the case of the pure torsion). The rationale of introducing a gradient term into the shear stress part is more reinforced if one notes that the non-effect of the shear stress gradient on fatigue interpreted by [7] is only found for some metals considered, but not meaning for all, thus such a presence of gradient is generally reasonable.

\section{Formulation of gradient multiaxial high-cycle fatigue criteria}

\subsection{General form of the classical fatigue endurance criteria}

A general form of the fatigue limit criteria can be written as follows:

$$
f\left(C_{a}\left(\boldsymbol{n}^{*}\right), N_{a}\left(\boldsymbol{n}^{*}\right), N_{m}\left(\boldsymbol{n}^{*}\right)\right) \leq 0
$$

$f$ is a function, chosen in many cases as linear; and $\boldsymbol{n}^{*}$ is the normal vector of the "critical plane"; and $C_{a}\left(\boldsymbol{n}^{*}\right), N_{a}\left(\boldsymbol{n}^{*}\right), N_{m}\left(\boldsymbol{n}^{*}\right)$ are the amplitudes of shear stress and normal stress, and the mean value of normal stress, respectively. The shear stress generally appears in fatigue criteria through its amplitude $C_{a}\left(\boldsymbol{n}^{*}\right)$, due to the independence of the fatigue limit with respect to the mean shear stress for a large number of metallic materials. And if one considers that the amplitude and the mean value of normal stress appear in form of their sum, i.e. $N_{\max }\left(\boldsymbol{n}^{*}\right)$, (7) can be rewritten:

$$
f\left(C_{a}\left(\boldsymbol{n}^{*}\right), N_{\max }\left(\boldsymbol{n}^{*}\right)\right)=C_{a}\left(\boldsymbol{n}^{*}\right)+a N_{\max }\left(\boldsymbol{n}^{*}\right)-b \leq 0
$$


with $a, b$ being two material parameters.

\subsection{General form of the stress gradient fatigue criteria}

The classical criteria (Crossland, Dang Van, ...) will now be modified to include the "size effect" 3 experimentally observed and beneficial influence of the stress gradient in the cases analyzed and corresponding to the surface fatigue and "decreasing stress gradient". At this stage it is reminded two crucial points. First, even if the torsion fatigue limit is generally independent from the shear stress gradient, it is not sure that it is also independent from the amplitude of the last. Second, the small pure size effect and the influence of the normal stress gradient on the bending fatigue limit show that adding only gradient terms could allow to model the fatigue tests results. Basing on these analyses, under multiaxial loading a generalization of the above experimental fact will be done.

With the presence of the unique gradient term (e.g. in $P_{\max }$ as Papadopoulos' proposal), the gradient fatigue criteria successfully represent the difference in the fatigue limit of uniaxial normal stress cyclic loadings, between fully reversed bending tests and fully reversed tension-compression tests. However, because of the vanishing of the gradient term of the model [7] in the case of pure torsion, such a formulation with the unique gradient term is not able to represent the possible influence of shear stress gradient amplitude and the "size effect" on the fatigue limit in torsion. For example, for torsion tests performed on specimen with various radii, the fatigue between the "reference test" (without any effect) and test at a certain radius is found identical using such an approach, which is contrary to experimental facts. The criterion adopted in [7] with only one stress gradient term in the normal stress part can describe gradient effects for tension-compression loadings with non zero hydrostatic stress, but not for shear stress loadings. Thus it leads to the necessity of adding a second gradient term to the shear stress part. Besides the stress gradient term appearing in the normal stress part in form of $G=\nabla \sigma_{k k}$, another gradient term, the gradient of stress tensor (or alternatively of deviatoric stress tensor) $\|\boldsymbol{Y}\|_{, a}=\|\nabla \boldsymbol{\sigma}\|_{, a}$, is added to the shear stress part. Basing on all these analyses a

$\overline{3}$ in the sense as discussed right from the introduction, actually it implies rather the gradient effect related to the size. 
new form of fatigue criteria taking into account gradient effects, is proposed:

$$
f\left(\widetilde{C_{a}}\left(\boldsymbol{n}^{*}\right), \widetilde{N}_{\max }\left(\boldsymbol{n}^{*}\right)\right)=\widetilde{C}_{a}\left(\boldsymbol{n}^{*}\right)+a \widetilde{N}_{\max }\left(\boldsymbol{n}^{*}\right)-b \leq 0
$$

where $\widetilde{C_{a}}\left(\boldsymbol{n}^{*}\right)$ and $\widetilde{N}_{\max }\left(\boldsymbol{n}^{*}\right)$ are extended definitions of the counterparts in the classical criteria. We propose the following forms for these quantities:

$$
\begin{gathered}
\widetilde{C_{a}}\left(\boldsymbol{n}^{*}\right)=C_{a}\left(\boldsymbol{n}^{*}\right) f_{c}\left(l_{\tau}^{*} \frac{\|\boldsymbol{Y}\|_{, a}}{C_{a}\left(\boldsymbol{n}^{*}\right)}\right) \\
\widetilde{N}_{\max }\left(\boldsymbol{n}^{*}\right)=N_{\max }\left(\boldsymbol{n}^{*}\right) f_{n}\left(l_{\sigma}^{*} \frac{\max _{t} \sum_{k=1}^{3} n_{i}^{*} n_{j}^{*} n_{k}^{*} Y_{i j k}}{N_{\max }\left(\boldsymbol{n}^{*}\right)}\right)
\end{gathered}
$$

The two functions $f_{c}$ and $f_{n}$ including the stress gradient terms, can have the following forms :

$$
\begin{gathered}
f_{c}\left(l_{\tau}^{*} \frac{\|\boldsymbol{Y}\|,_{a}}{C_{a}\left(\boldsymbol{n}^{*}\right)}\right)=\sqrt{1-\left(l_{\tau}^{*} \frac{\|\boldsymbol{Y}\|_{a}}{C_{a}\left(\boldsymbol{n}^{*}\right)}\right)^{n_{\tau}}} \\
f_{n}\left(l_{\sigma}^{*} \frac{\max _{t} \sum_{k=1}^{3} n_{i}^{*} n_{j}^{*} n_{k}^{*} Y_{i j k}}{N_{\max }\left(\boldsymbol{n}^{*}\right)}\right)=\sqrt{1-\left(l_{\sigma}^{*} \frac{\max _{t} \sum_{k=1}^{3} n_{i}^{*} n_{j}^{*} n_{k}^{*} Y_{i j k}}{N_{\max }\left(\boldsymbol{n}^{*}\right)}\right)^{n_{\sigma}}}
\end{gathered}
$$

Note that $f_{c}$ could alternatively be function of the gradient of the stress deviator. These expressions will be specified for the two criteria considered in the next sections. $l_{\tau}^{*}$ and $l_{\sigma}^{*}$ are two material characteristic lengths; $n_{\tau}$ and $n_{\sigma}$ are two material characteristic exponents, or actually gradient-amplifying exponents introduced to get a more flexibility in capturing any large experimental data class.

To sum up, it is clear to confirm the necessity of the simultaneous presence of the two gradient terms in fatigue criteria, one for the normal stress part through $G$ and the other for the shear stress part through $\|Y\|_{, a}$. These criteria are used to describe fatigue limits under different kinds of loading (loading effect) in which the gradient effect is taken into account and the pure size effect is insignificant compared to the other. After all, using as a basis the classical fatigue criteria for formulating the stress gradient dependent fatigue criteria after the above methodology, some illustrations will be made in the following, one for Crossland criterion and the other for Dang Van criterion. The same approach could be in principle applied to other classical fatigue criteria. 


\section{Gradient Crossland criterion}

\subsection{Classical Crossland criterion}

The Crossland criterion [29] is used as a basis for the development of a gradient dependent criterion. The classical Crossland criterion defines the fatigue limit of metallic specimens subjected to multiaxial in-phase cyclic stress states as, $c f$. [31]:

$$
{\sqrt{J_{2}}}_{, a}+\alpha_{c} P_{\max } \leq \gamma_{c}
$$

where $\alpha_{c}$ and $\gamma_{c}$ are material parameters, $\sqrt{J_{2}, a}$ is the amplitude of the square root of the second invariant of the stress deviator tensor and $P_{\max }$ is the maximum hydrostatic stress during a loading cycle.

The amplitude of the square root of the second invariant of the stress deviator can be defined, in general case, as the half-length of the longest chord of the deviatoric stress path by :

$\sqrt{J_{2}, a}=\frac{1}{2 \sqrt{2}} \max _{t_{1}}\left\{\max _{t_{2}}\left\|\boldsymbol{s}\left(t_{2}\right)-\boldsymbol{s}\left(t_{1}\right)\right\|\right\}=\frac{1}{2 \sqrt{2}} \max _{t_{1}}\left\{\max _{t_{2}} \sqrt{\left(\boldsymbol{s}\left(t_{2}\right)-\boldsymbol{s}\left(t_{1}\right)\right):\left(\boldsymbol{s}\left(t_{2}\right)-\boldsymbol{s}\left(t_{1}\right)\right)}\right\}$

or as the radius of the smallest hypersphere circumscribed to the deviatoric stress path by :

$$
\sqrt{J_{2}, a}=\frac{1}{\sqrt{2}} \min _{\boldsymbol{s}_{1}}\left\{\max _{t}\left\|\boldsymbol{s}(t)-\boldsymbol{s}_{1}\right\|\right\}=\frac{1}{\sqrt{2}} \min _{\boldsymbol{s}_{1}}\left\{\max _{t} \sqrt{\left(\boldsymbol{s}(t)-\boldsymbol{s}_{1}\right):\left(\boldsymbol{s}(t)-\boldsymbol{s}_{1}\right)}\right\}
$$

The maximum value that the hydrostatic stress reaches during the loading cycle is:

$$
P_{\text {max }}=\max _{t}\left\{\frac{1}{3} \operatorname{tr}(\sigma(t))\right\}=\frac{\sigma_{k k}}{3}
$$

In these equations, the summation convention over repeated indices holds and $s$ and $p$ are respectively the deviatoric and spherical part of the stress tensor:

$$
p(t)=\frac{1}{3} \operatorname{tr}(\sigma(t)) \quad \text { and } \quad \boldsymbol{s}(t)=\boldsymbol{\sigma}(t)-p(t) \boldsymbol{I}
$$

and $\boldsymbol{I}$ is the second order unit tensor.

The material parameters $\alpha_{c}$ and $\gamma_{c}$ can be related to the fully reversed tensioncompression fatigue limit, denoted by $s_{r e f}$, and to the torsion fatigue limit, denoted 
by $\mathrm{t}_{r e f}$, by:

$$
\gamma_{c}=\mathrm{t}_{r e f} ; \quad \alpha_{c}=\frac{3 \mathrm{t}_{r e f}}{s_{r e f}}-\sqrt{3}
$$

As well-known, to obtain the observed detrimental effect of a tensile mean stress state, the parameter $\alpha_{c}$ in Eq. (19), must be positive, and therefore: $\mathrm{t}_{r e f}>s_{\text {ref }} / \sqrt{3}$. Furthermore, since the "size" and gradient effects are not captured in the classical Crossland criterion, it is only valid for the specimen large enough and smooth enough. For this reason, the subscript "ref" used for the fatigue limits $s_{r e f}$ and $\mathrm{t}_{r e f}$ means material constants independent of the "size" and gradient effects which will be used as references for other case-studies. Concretely, in the case where these effects could be important, new fatigue criteria to include them are required. As well for this reason, in Eq. (19) just $\mathbf{S}_{r e f}$ is chosen instead of $\mathbf{f}$ because in size range under consideration where the gradient effects can be significant, just $\mathbf{S}_{\mathbf{r e f}}$ is regarded as a characteristic constant intrinsic to material but not $\mathbf{f}$ in the sense that only that is exempt from the gradient effect.

\subsection{Formulation of the gradient Crossland criterion}

Using as a basis the classical Crossland criterion, Eq. (14) and the general framework for the development of a gradient dependent fatigue limit criterion (Eq. 9), a new version can be written in the form:

$$
\sqrt{\widetilde{J}_{2, a}}+\alpha_{g} \widetilde{P_{\max }} \leq \gamma_{g}
$$

From the classical expression of $\sqrt{J_{2}}$, a new form embedded with gradient term is proposed:

$$
\sqrt{\widetilde{J}_{2}}=\sqrt{\frac{1}{2}\|\boldsymbol{s}\|^{2}\left[1-\left(l_{\tau} \frac{\|\boldsymbol{Z}\|}{\|s\|}\right)^{n_{\tau}}\right]}=\sqrt{J_{2}} \sqrt{1-\left(l_{\tau} \frac{\|\boldsymbol{Z}\|}{\|\boldsymbol{s}\|}\right)^{n_{\tau}}}
$$

$l_{\tau}$ is a material characteristic length, and $n_{\tau}$ is a material characteristic exponent. The quantity $\|\boldsymbol{Z}\|=\|\nabla \boldsymbol{s}\|$ is used as an indicator of the influence of the gradient of the stress deviator which reflects the spatial non-uniform distribution of stress state. Similarly to [7], the ratio of the norm $\|Z\|$ over the norm $\|s\|$ is called reduced gradient too. However in the current work that is more exactly understood as the shear reduced gradient of the new fatigue criterion. 
Eq. (21) can be found in more familiar and visible way when setting $n_{\tau}=2$ :

$$
\sqrt{\widetilde{J_{2}}}=\sqrt{\frac{1}{2}\left[\|s\|^{2}-l_{\tau}^{2}\|\boldsymbol{Z}\|^{2}\right]}
$$

which is similar to the expression of plasticity criteria within the framework of gradient dependent models, see e.g. [34]. However, the present study will not fix $n_{\tau}=2$ but let it be a material parameter to calibrate experimentally.

In the spirit of Eq. (22), and taking account of the recent proposition of Amargier et al [1] which expression includes the product of $\sqrt{J_{2}, a}$ and a function of the hydrostatic stress gradient, we define the following amplitude $\sqrt{\widetilde{J}_{2, a}}$ which combines $\sqrt{J_{2, a}}$ and the full stress gradient $\|\boldsymbol{Y}\|_{, a}$ is the form:

$$
\sqrt{\widetilde{\widetilde{J}}_{2, a}}=\sqrt{J_{2, a}} \sqrt{1-\left(l_{\tau} \frac{\|\boldsymbol{Y}\|_{, a}}{\|\boldsymbol{s}\|_{, a}}\right)^{n_{\tau}}}
$$

For the sake of illustration, the following treatment is performed for in-phase loading where simple expressions can be obtained. The stress state at a point is written as:

$$
\sigma_{i j}(t)=\widehat{\sigma}_{i j} \sin (\omega t)+\bar{\sigma}_{i j}, \quad i, j=x, y, z,
$$

where $\widehat{\sigma}_{i j}$ is the amplitude of the $(i j)$ stress component oscillating around a $\bar{\sigma}_{i j}$ mean value and over T- the loading period.

The expression of the third order tensor $\boldsymbol{Y}$ and the amplitude of its norm $\|\boldsymbol{Y}\|_{, a}$ are elaborated in the present case-study, as Eq. (25) or (26):

$$
\begin{aligned}
\boldsymbol{Y}(t) & =\nabla \boldsymbol{\sigma}(t) \Longrightarrow Y_{i j k}(t)=\sigma_{i j, k}(t)=\widehat{\sigma}_{i j, k} \sin (\omega t)+\bar{\sigma}_{i j, k}, \quad i, j, k=x, y, z, \\
\|\boldsymbol{Y}\|_{, a} & =\min _{\boldsymbol{Y}_{1}}\left\{\max _{t}\left\|\boldsymbol{Y}(t)-\boldsymbol{Y}_{1}\right\|\right\}=\min _{\boldsymbol{Y}_{1}}\left\{\max _{t} \sqrt{\left(\boldsymbol{Y}(t)-\boldsymbol{Y}_{1}\right) \bullet\left(\boldsymbol{Y}(t)-\boldsymbol{Y}_{1}\right)}\right\} \\
\text { or }\|\boldsymbol{Y}\|_{, a} & =\max _{t_{1}}\left\{\max _{t_{2}} \sqrt{\left(\boldsymbol{Y}\left(t_{2}\right)-\boldsymbol{Y}\left(t_{1}\right)\right) \bullet\left(\boldsymbol{Y}\left(t_{2}\right)-\boldsymbol{Y}\left(t_{1}\right)\right)}\right\}=\sqrt{4 \widehat{Y}_{i j k} \widehat{Y}_{i j k}}
\end{aligned}
$$

with $\widehat{Y}_{i j k}=\widehat{\sigma}_{i j, k}$, and the product definition: $\boldsymbol{Y} \bullet \boldsymbol{Y}=Y_{i j k} Y_{i j k}$.

Thus, from Eq. (16) with the expression of $\sqrt{J_{2, a}}$ and of $\|s\|_{, a}=\sqrt{4 \widehat{s}_{i j} \widehat{s}_{i j}}$, and Eq. 
(25) with the expression of $\|Y\|_{, a}, \sqrt{{\widetilde{J_{2}}}_{, a}}$ is elaborated as Eq. (23):

$$
\sqrt{\widetilde{J}_{2, a}}=\sqrt{\frac{1}{2} \widehat{s}_{i j} \widehat{s}_{i j}\left[1-\left(l_{\tau} \frac{\sqrt{\widehat{Y}_{i j k} \widehat{Y}_{i j k}}}{\sqrt{\widehat{s}_{i j} \widehat{s}_{i j}}}\right)^{n_{\tau}}\right]}
$$

With respect to $\widetilde{P_{\max }}$, the same form as the one of [7] is proposed:

$$
\widetilde{P_{\max }}=P_{\max }\left[1-\left\langle l_{\sigma} \frac{\|\boldsymbol{G}\|}{P_{\max }}\right\rangle^{n_{\sigma}}\right]
$$

with $G$, the gradient of $P_{\max }$ being the vector:

$$
\boldsymbol{G}=\nabla P_{\max }={ }^{T}\left[P_{\max , x}, P_{\max , y}, P_{\max , z}\right]
$$

which norm $\|\boldsymbol{G}\|$ is:

$$
\|\boldsymbol{G}\|=\sqrt{\left(P_{\max , x}\right)^{2}+\left(P_{\max , y}\right)^{2}+\left(P_{\max , z}\right)^{2}}
$$

The norm of the gradient of $P_{\max }$, i.e. $\|\boldsymbol{G}\|$, is used as an indicator of the influence of the normal stresses gradient. One more again, the ratio of the norm $\|\boldsymbol{G}\|$ over $P_{\text {max }}$ is called here hydrostatic reduced gradient.

Moreover, in Eq. (28), $l_{\sigma}$ and $n_{\sigma}$ are also material characteristic parameters with the same signification as $l_{\tau}$ and $n_{\tau}$. as in [7], to avoid the degradation in the case of null value of $P_{\max }$ but non-zero value of its gradient, an extended definition of the McCauley bracket $\langle\circ\rangle$ is adopted:

$$
\begin{aligned}
& \left\langle l_{\sigma} \frac{\|\boldsymbol{G}\|}{P_{\max }}\right\rangle=l_{\sigma} \frac{\|\boldsymbol{G}\|}{P_{\max }} \text { if } l_{\sigma} \frac{\|\boldsymbol{G}\|}{P_{\max }}>0, \text { and }\left\langle l_{\sigma} \frac{\|\boldsymbol{G}\|}{P_{\max }}\right\rangle=0 \text { if } l_{\sigma} \frac{\|\boldsymbol{G}\|}{P_{\max }} \leq 0 \\
& \left\langle l_{\sigma} \frac{\|\boldsymbol{G}\|}{P_{\max }}\right\rangle=0 \text { if } P_{\max }=0
\end{aligned}
$$

The properties expressed by Eq. (31) have been used to deliberately neglect the gradient effect in the case of a fully compressive cycle of the hydrostatic stress (i.e. $\left.P_{\max }<0\right)$. This assumption can be disregarded if experimental facts show that it is irrelevant. 
Finally, the criteria written as:

$$
\sqrt{J_{2}} \sqrt{1-\left(l_{\tau} \frac{\|\boldsymbol{Y}\|_{, a}}{\|\boldsymbol{s}\|_{, a}}\right)^{n_{\tau}}}+\alpha_{g} P_{\max }\left[1-\left\langle l_{\sigma} \frac{\|\boldsymbol{G}\|}{P_{\max }}\right\rangle^{n_{\sigma}}\right]-\gamma_{g}<0
$$

has six materials parameters $\left(\alpha_{g}, \gamma_{g}, l_{\tau}, l_{\sigma}, n_{\tau}, n_{\sigma}\right)$ to be identified experimentally.

\subsection{Calibration of the material parameters}

As the proposed criterion reduces to the classical Crossland one in the absence of "size" and gradient effects, the parameters $\alpha_{g}$ et $\gamma_{g}$ are the same as those in the classical version, and given by $\gamma_{g}=\gamma_{c}=\mathrm{t}_{r e f}$, and $\alpha_{g}=\alpha_{c}=\frac{3 \mathrm{t}_{r e f}}{s_{r e f}}-\sqrt{3}$.

A procedure for obtaining the parameters from fully reversed torsion and fully reversed constant moment bending tests is detailed hereafter.

\section{a) Fully reversed torsion tests}

The criterion described by Eq. (20) is applied, first, to the case of fully reversed torsion tests. Let us denote by $\mathrm{t}(R)$ the fatigue limit of a specimen of radius $R$. Considering the critical points (located at $r= \pm R$ ), their relevant quantities are:

$$
\begin{gathered}
\boldsymbol{\sigma}=\widehat{\sigma}_{x \theta} \sin (\omega t)\left(e_{x} \otimes e_{\theta}+e_{\theta} \otimes e_{x}\right)=\mathrm{t}(R) \sin (\omega t)\left(e_{x} \otimes e_{\theta}+e_{\theta} \otimes e_{x}\right) \\
\widehat{s}_{i j} \widehat{s}_{i j}=2(\mathrm{t}(R))^{2} \text { and } \widehat{Y}_{i j k} \widehat{Y}_{i j k}=2\left(\frac{\widehat{\sigma}_{x \theta}}{R}\right)^{2}=2\left(\frac{\mathrm{t}(R)}{R}\right)^{2} \\
\widetilde{P_{\max }}=0 \text { and } \sqrt{\widetilde{J}_{2, a}}=\mathrm{t}(R) L_{\tau}(R) \\
\text { with } L_{\tau}(R)=\sqrt{1-\left(l_{\tau} / R\right)^{n_{\tau}}} \text { : shear reduced gradient }
\end{gathered}
$$

And using Eqs. (33) the proposed fatigue criterion, Eq. (20), leads to:

$$
\mathrm{t}(R)=\frac{\mathrm{t}_{\text {ref }}}{\sqrt{1-\left(l_{\tau} / R\right)^{n_{\tau}}}}
$$

This formula is used to calibrate the three material parameters $\tau_{r e f}, l_{\tau}$ and $n_{\tau}$, using the experiment curve relating the fatigue limit $\mathrm{t}(R)$ to the radius of the specimen. The material parameters are then calibrated using the least square method on the tests points; and therefore the optimal parameters (i.e. the values which minimize the scatter between the predicted and experimental points) for the criterion are obtained. As an illustration, the torsion fatigue tests given by Massonnet [27] are used 
to identify $\tau_{r e f}, l_{\tau}$ and $n_{\tau}$, as shown in Fig. 6. A visual image of $\mathrm{t}_{r e f}$ aforementioned is as well found in this graph. The values obtained are: $\tau_{\text {ref }}=115 \mathrm{MPa}$, $l_{\tau}=1.6 \mathrm{~mm}$ and $n_{\tau}=0.5$.

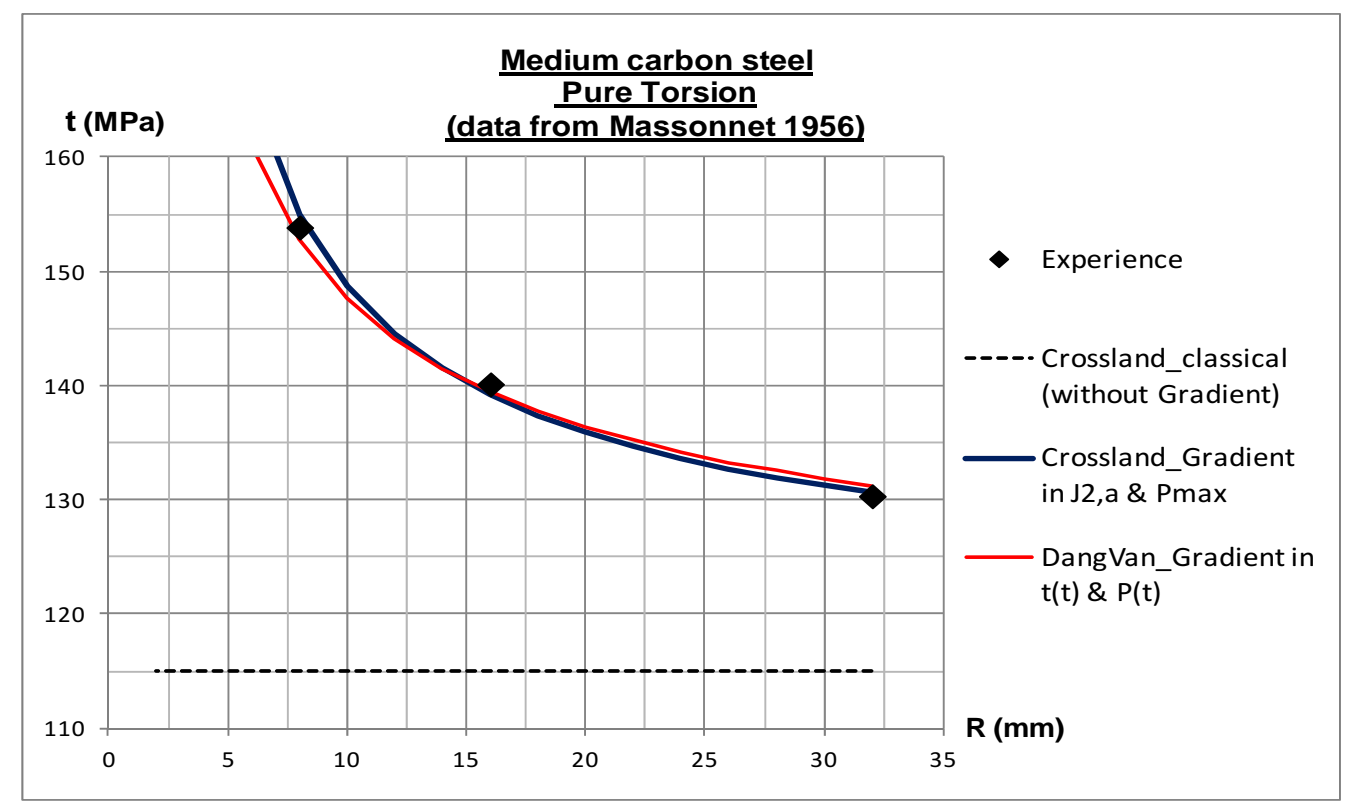

Fig. 6. Fully reversed torsion fatigue limit of smooth cylindrical samples (cf. Massonnet [28])

We notice that the fatigue limit tends toward infinity as the radius tends toward the characteristic length $l_{\tau}$. It defines the limit of the model. Nevertheless, it indicates a tendency consistent with the fact "Smaller is Stronger".

\section{b) Fully reversed constant moment bending tests (four-point bending tests)}

To calibrate the other parameters $\left(l_{\sigma}, n_{\sigma}\right)$ the criterion Eq. (20) is now applied to the case of fully reversed four-point bending tests. The fatigue limit of a specimen of radius $R$ is denoted $\mathrm{f}(R)$. Considering the most stressed points, i.e. points lying at $L \leq x \leq L+l$ and at $y= \pm R$, relevant quantities, in particular $\sqrt{\widetilde{J}_{2}, a}$, calculated by Eq. (27) are given by:

$$
\begin{gathered}
\boldsymbol{\sigma}=\sigma_{x x} e_{x} \otimes e_{x}=\widehat{\sigma}_{x x} \sin (\omega t) e_{x} \otimes e_{x}=\mathrm{f}(R) \sin (\omega t) e_{x} \otimes e_{x} \\
{\sqrt{\widetilde{J}_{2, a}}}=\frac{\mathrm{f}(R)}{\sqrt{3}} L_{\tau f}(R)
\end{gathered}
$$




$$
\text { with } \quad L_{\tau f}(R)=\sqrt{1-(3 / 2)^{n_{\tau} / 2}\left(l_{\tau} / R\right)^{n_{\tau}}}=L_{\tau f}\left(L_{\tau}\right)
$$

Similarly, with the help of Eq. (28), $\widetilde{P_{\max }}$ can be elaborated, in this case-study, as:

$$
P_{\max }=\frac{\widehat{\sigma}_{x x}}{3}=\frac{\mathrm{f}(R)}{3} \quad \text { and } \quad \widetilde{P_{\max }}=\frac{\mathrm{f}(R)}{3} L_{\sigma f}(R)
$$

with $L_{\sigma f}(R)=1-\left(l_{\sigma} / R\right)^{n_{\sigma}}:$ normal reduced gradient

and $\quad \boldsymbol{G}=\left[P_{\max , x}=0, P_{\max , y}=\frac{\widehat{\sigma}_{x x, y}}{3}=\frac{\widehat{F} L}{3 I}=\frac{\widehat{\sigma}_{x x}}{3 R}=\frac{\mathrm{f}}{3 R}, P_{\max , z}=0\right]$

$$
\text { and } \quad\|\boldsymbol{G}\|=\frac{\mathrm{f}}{3 R}
$$

Finally, the fatigue criterion (20), lead to the following expression of the fatigue limit f:

$$
\mathrm{f}(R)=\frac{s_{r e f}}{1-l_{\sigma} R^{-n_{\sigma}}\left(1-\frac{s_{r e f}}{\sqrt{3} \mathrm{t}_{\text {ref }}}\right)-\frac{s_{r e f}}{\sqrt{3} \mathrm{t}_{\text {ref }}}\left(1-L_{\tau f}(R)\right)} \geq s_{\text {ref }}
$$

As previously, this formula is used to calibrate the three material parameters $s_{\text {ref }}$, $l_{\sigma}$ and $n_{\sigma}$, using the experiment curve relating the fatigue limit $\mathrm{f}(R)$ to the radius of the specimen. The material parameters are calibrated using the least square method on the tests points to obtain the optimal parameters. As an illustration, the fourpoint bending tests given by Pogoretskii [14] are used to identify $s_{r e f}, l_{\sigma}$ and $n_{\sigma}$ assuming that $\mathrm{t}_{r e f}$ and $L_{\tau f}(R)$ are known from the previous calibration. The result is shown in Fig. 7d. A visual image of $\mathrm{s}_{\text {ref }}$ aforementioned is as well found in this graph.

\section{c) Application to the fully reversed cantilever bending tests}

It is of more interest to apply the criterion Eq. (20) to the case of fully reversed cantilever bending tests to see, besides the well-known role of $R$, the role of $L$. The difference and similarity in fatigue limit between two kinds of bending, i.e. fourpoint bending and cantilever bending is analyzed. Let us denote the corresponding fatigue limit $f^{\prime}(R)$. Considering the most stressed points, i.e. points at $x=0$ and at $y= \pm R$ (Fig. 3), again, respective quantities and then $\sqrt{\widetilde{J}_{2_{, a}}}$ determined by Eq. (27) are given:

$$
\boldsymbol{\sigma}=\sigma_{x x} e_{x} \otimes e_{x}=\widehat{\sigma}_{x x} \sin (\omega t) e_{x} \otimes e_{x}=f^{\prime} \sin (\omega t) e_{x} \otimes e_{x}
$$




$$
\begin{gathered}
\sqrt{\widetilde{\widetilde{J}}_{2, a}}=\frac{f^{\prime}(R)}{\sqrt{3}} L_{\tau f^{\prime}}(R, L) \\
\text { with } L_{\tau f^{\prime}}(R, L)=\sqrt{1-(3 / 2)^{n_{\tau} / 2}\left(l_{\tau} / R\right)^{n_{\tau}}\left(1+\frac{R^{2}}{L^{2}}\right)^{n_{\tau} / 2}}
\end{gathered}
$$

Similarly, with the help of Eq. (28), $\widetilde{P_{\max }}$ can be evaluated for this case:

$$
\begin{aligned}
& \boldsymbol{G}=\left[P_{\max , x}=\frac{\widehat{\sigma}_{x x, x}}{3}=\frac{-f^{\prime}(R)}{3 L}, P_{\text {max }, y}=\frac{\widehat{\sigma}_{x x, y}}{3}=\frac{f^{\prime}(R)}{3 R}, P_{\text {max }, z}=0\right] \\
& \|\boldsymbol{G}\|=\frac{f^{\prime}(R)}{3 R} \sqrt{1+\frac{R^{2}}{L^{2}}} \\
& \widetilde{P_{\text {max }}}=\frac{f^{\prime}(R)}{3} L_{\sigma f^{\prime}}(R, L) \\
& \text { with } L_{\sigma f^{\prime}}(R, L)=1-\left(l_{\sigma} / R\right)^{n_{\sigma}}\left(1+\frac{R^{2}}{L^{2}}\right)^{n_{\tau} / 2}
\end{aligned}
$$

Finally, from Eq. (20), $f^{\prime}$ is obtained as:

Using the Eq. (50), a class of experimental data of the cantilever bending fatigue tests are successfully reproduced, as shown in Fig. 7(a-c).

On the other hand, for specimens with $R \ll L$, the ratio $\left(R^{2} / L^{2}\right)$ is negligible. Under these circumstances the fatigue limit in fully reversed constant moment and cantilever bending of specimens of the same radius, coincide and are related to the tension-compression fatigue limit by Eq. (42). Using this assumption an important number of bending fatigue limits has been analyzed. It turned out that the value $1 / 2$ for the exponents $n_{\tau}$ and $n_{\sigma}$ brought adequate predictions for the experiments studied. The criterion is then:

$$
\sqrt{J_{2}} \sqrt{1-\left(l_{\tau} \frac{\|\boldsymbol{Y}\|_{, a}}{\|s\|_{, a}}\right)^{1 / 2}}+\alpha_{g} P_{\max }\left[1-\sqrt{l_{\sigma}\left\langle\frac{\|\boldsymbol{G}\|}{P_{\max }}\right\rangle}\right]-\gamma_{g}<0
$$

with four materials parameters $\left(\alpha_{g}, \gamma_{g}, l_{\tau}, l_{\sigma}\right)$ to be identified experimentally.

Figure 7 shows some test results of rotating bending fatigue limits from the literature in which the fatigue limits are plotted against the specimen radii. Figures $7(a-c)$ are related to cantilever bending tests and Fig. 7(d) depicts constant moment tests. 
The solid curves in these graphs present the simulation with the proposed criterion, where the value $n=1 / 2$ has been assumed. As shown, the accordance with the experimental data is satisfactory.

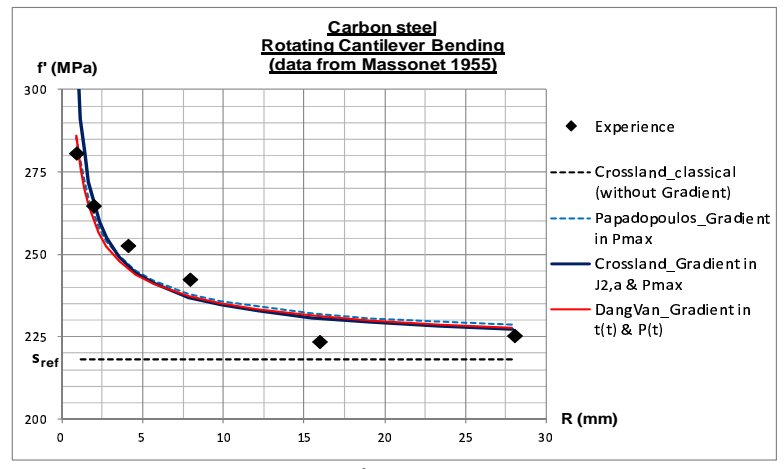

a)

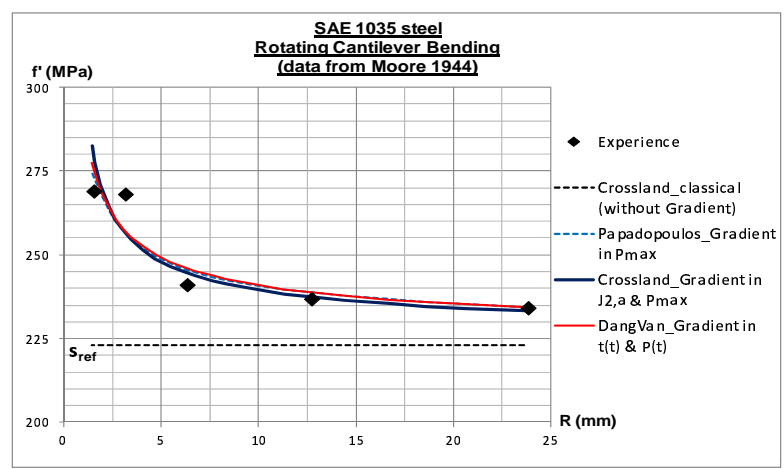

c)

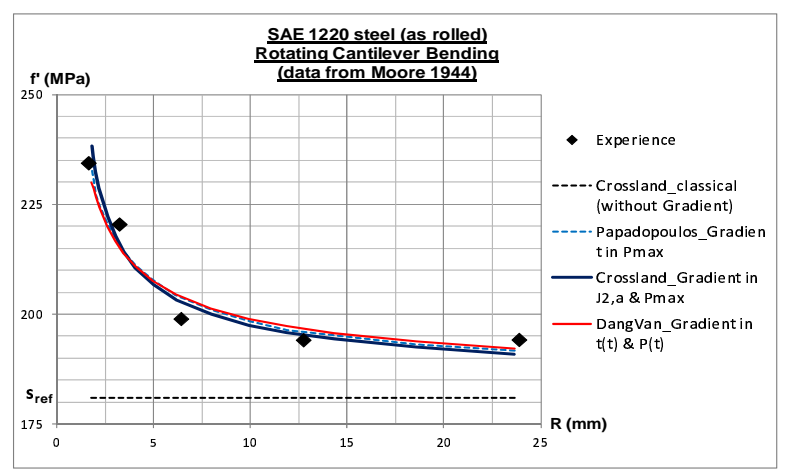

b)

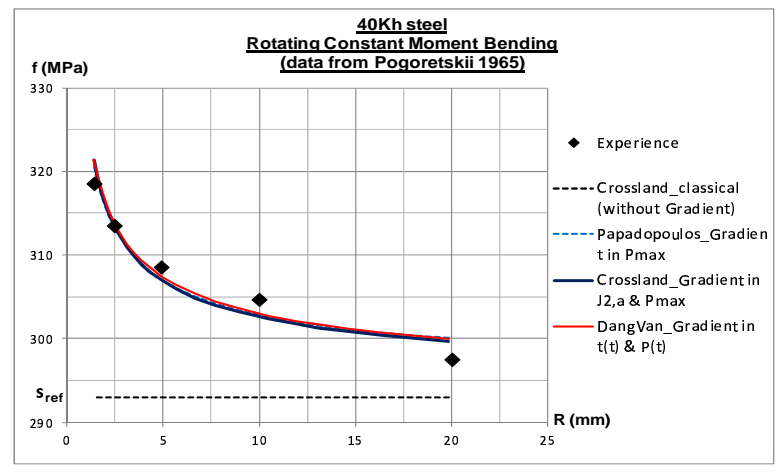

d)

Fig. 7. Fully reversed bending fatigue limits of cylindrical specimen (Massonnet [27],

Moore \& Morkovin [33], Pogoretskii \& Karpenko [14], Papadopoulos \& Panoskaltsis [7])

\section{d) Application to the fully reversed combined bending-twisting tests}

The criterion (20) is now applied to the case of fully reversed in-phase bending and torsion fatigue tests [7]. Specimens of toroidal shape are usually used for these tests. Considering the most stressed points, i.e. points at $y= \pm R, z=0$ and denoting by $\sigma_{a}$ and $\tau_{a}$ the limit amplitudes of the normal and shear stresses respectively, related quantities especially $\sqrt{\widetilde{J}_{2}}$, , by Eq. (27), are given:

$$
\begin{gathered}
\boldsymbol{\sigma}=\sigma_{a} \sin (\omega t) e_{x} \otimes e_{x}+\tau_{a} \sin (\omega t)\left(e_{x} \otimes e_{\theta}+e_{\theta} \otimes e_{x}\right) \\
{\sqrt{\widetilde{J}_{2}}}=\sqrt{\frac{\sigma_{a}^{2}}{3}+\tau_{a}^{2}} L_{\tau c}\left(\sigma_{a}, \tau_{a}, R\right)
\end{gathered}
$$


with:

$$
L_{\tau c}\left(\sigma_{a}, \tau_{a}, R\right)=\sqrt{1-\left(l_{\tau c} / R\right)^{n_{\tau}}} \quad \text { with } \quad l_{\tau c}=l_{\tau}^{n_{\tau}}\left(\frac{3 \sigma_{a}^{2}+6 \tau_{a}^{2}}{2 \sigma_{a}^{2}+6 \tau_{a}^{2}}\right)^{n_{\tau} / 2}
$$

For the maximum hydrostatic stress $\widetilde{P_{\max }}$, the same expression as the case of bending tests, Eq. (38), is here given:

$$
\widetilde{P_{\max }}=\frac{\sigma_{a}}{3} L_{\sigma c}(R) \quad \text { with } \quad L_{\sigma c}=1-\left(l_{\sigma} / R\right)^{n_{\sigma}}=L_{\sigma f}
$$

The criterion is therefore expressed as:

$$
\begin{aligned}
& \sqrt{\left(\frac{\sigma_{a}^{2}}{3}+\tau_{a}^{2}\right)} L_{\tau c}+\alpha_{g} \frac{\sigma_{a}}{3} L_{\sigma c}<\gamma_{g}, \quad \text { or more concretely, } \\
& \sqrt{\left(\frac{\sigma_{a}^{2}}{3}+\tau_{a}^{2}\right)} \sqrt{1-\left(l_{\tau} / R\right)^{n_{\tau}}\left(\frac{3 \sigma_{a}^{2}+6 \tau_{a}^{2}}{2 \sigma_{a}^{2}+6 \tau_{a}^{2}}\right)^{n_{\tau} / 2}}+\alpha_{g} \frac{\sigma_{a}}{3}\left(1-\left(l_{\sigma} / R\right)^{n_{\sigma}}\right)<\gamma_{g}
\end{aligned}
$$

\section{Comparison with classical and Papadopoulos results}

The application of the classical Crossland criterion in the case of fully reversed inphase combined tension-compression and torsion fatigue tests gives the following "ellipse arc equation":

$$
\left(\frac{\tau_{a}}{\mathrm{t}_{\text {ref }}}\right)^{2}+\left(\frac{2 s_{\text {ref }}}{\sqrt{3} \mathrm{t}_{\text {ref }}}-1\right)\left(\frac{\sigma_{a}}{s_{\text {ref }}}\right)^{2}+\left(2-\frac{2 s_{r e f}}{\sqrt{3} \mathrm{t}_{r e f}}\right)\left(\frac{\sigma_{a}}{s_{r e f}}\right) \leq 1
$$

which delimits in the $\sigma_{a}-\tau_{a}$ plane the safe domain. Eq. (56) shows high discrepancies between predictions and experiments for the fatigue limit in combined bending-twisting with the "size" and gradient effects (Fig. 8). As in [7], to bypass this trouble, modified material parameters $\alpha_{g}$ and $\gamma_{g}$ related to the bending fatigue limit $\mathrm{f}(R)$ and torsion fatigue limit $\mathrm{t}(R)$ (instead of $s_{\text {ref }}$ and $\mathrm{t}_{\text {ref }}$ ), experimentally determined on specimens (radius $R$ ) of the same geometry as used for the combined tension-compression and torsion tests, can be used. Two things different from [7] are, first, the use of $\mathrm{f}(R)$ and $\mathrm{t}(R)$ determined at the specific radius $R$ of specimens under consideration, and second, the substitution of both $s_{\text {ref }}$ and $\mathrm{t}_{\text {ref }}$ by $\mathrm{f}(R)$ and $\mathrm{t}(R)$, instead of only one $s_{\text {ref }}$ by $\mathrm{f}$. Then $\gamma_{g}=\mathrm{t}(R)$ and $\alpha_{g}=\frac{3 \mathrm{t}(R)}{f(R)}-\sqrt{3}$, and the 
application of the Crossland criterion using these new values of $\alpha_{g}$ and $\gamma_{g}$ leads to the new ellipse arc equation:

$$
\left(\frac{\tau_{a}}{\mathrm{t}(R)}\right)^{2}+\left(\frac{2 \mathrm{f}(R)}{\sqrt{3} \mathrm{t}(R)}-1\right)\left(\frac{\sigma_{a}}{\mathrm{f}(R)}\right)^{2}+\left(2-\frac{2 \mathrm{f}(R)}{\sqrt{3} \mathrm{t}(R)}\right)\left(\frac{\sigma_{a}}{\mathrm{f}(R)}\right) \leq 1
$$

It is noticed that this formula is very similar to the well known ellipse arc formula of Gough and Pollard.

In the following, we show that Eq. (55) obtained with the proposed criterion, reduces to Eq. (57) for certain values of the material parameters. Indeed, first let us review the constant moment bending case. Assuming $n_{\tau}=0.5$ as validated by a large number of experiment, Eq. (37) yields:

$$
L_{\tau f}(R) \approx \sqrt{1-\left(l_{\tau} / R\right)^{n_{\tau}}}=L_{\tau}
$$

Resulting from Eqs. $(35,34)$ and $(42,39)$, the expressions of $L_{\tau}$ and $L_{\sigma f}$ are reported below for completeness:

$$
L_{\tau}=\frac{\mathrm{t}_{r e f}}{\mathrm{t}} ; \quad L_{\sigma f}=\frac{1 / \mathrm{f}-1 / \sqrt{3} \mathrm{t}}{1 / s_{r e f}-1 / \sqrt{3} \mathrm{t}_{r e f}}
$$

And second, consider now again the combined bending-twisting case by evaluating the function $h\left(\tau_{a}\right)=\left(\frac{3 \sigma_{a}^{2}+6 \tau_{a}^{2}}{2 \sigma_{a}^{2}+6 \tau_{a}^{2}}\right)^{n_{\tau} / 2}$ to get an approximation for $L_{\tau c}$ and $l_{\tau c}$ defined in Eq. (53). Again, for $n_{\tau}=0.5$ assumed before, $h\left(\tau_{a}\right)$ is in the interval $[1,1.1)$, we take $h\left(\tau_{a}\right) \approx 1$, so that $l_{\tau c} \approx l_{\tau}^{n_{\tau}}$. Therefore,

$$
L_{\tau c}(R)=\sqrt{1-\left(l_{\tau c} / R\right)^{n_{\tau}}} \approx L_{\tau}
$$

Finally, replacing $L_{\tau c}$ and $L_{\sigma c}$ in Eq. (55) by their approximations, with the help of Eq. (59), Eq. (57), is recovered.

In Fig. 8 the test results of bending-twisting conducted by [30] on $S A E 4340$ steel, are depicted. In the same figure, the Crossland analytical ellipse arc based on the $s_{r e f}-\mathrm{t}_{r e f}$ fatigue limits, Eq. (56), is plotted too. As we can see, all the test points fall considerably outside this analytical ellipse arc. This demonstrates the effect of the normal stress gradient, as the analytical ellipse arc (Eq. 56) is obtained with 
zero normal stress gradient, whereas the experimental data for combined bendingtwisting tests have a non-zero stress gradient. Furthermore, it is interesting to reconsider some analyses of [7] when stated that "the higher the normal stress due to bending, the higher the difference between test points and Crossland ellipse arc, whereas the higher the shear stress, the smaller the difference between test points and Crossland ellipse arc becomes...". First, the difference between test points and classical Crossland ellipse arc near the X-axis where the normal load is predominant, is a proof of the beneficial "size" and gradient effects. Indeed, the difference between two kinds of fatigue test can be clearly seen: the bending test (test points) includes the beneficial effects of the normal stress gradient; the tensioncompression test (Crossland ellipse arc) excludes these effects due to the gradientfree stress state. Second, the coincidence between test points and Crossland ellipse arc near the $y$-axis with predominant shear stress is actually natural due to the fact that $\mathrm{t}_{r e f}$ used to depict the Crossland ellipse arc after Eq. (56) and corresponding test point on the $\mathrm{y}$-axis are actually the same, thus this coincidence really does not reflect the "lack of sensitivity of the limiting fatigue stress on the gradient of the shear stress" [7] due to the fact that the "size" and gradient effects in torsion test were not accounted for. Third, to account for the "size" and shear gradient amplitude effect, a clear distinction must be made between $\mathrm{t}_{\text {ref }}$ determined at the radius $R_{\infty}$ of specimen large enough and $\mathrm{t}(R)$ determined at the radius $R$ of the considered specimen. Then all these above analyses affirm, first, the "size effect" on fatigue limits (Smaller is Stronger) as well as the beneficial effect of the normal stress gradient (Higher Gradient is Stronger), and second, the necessity of a distinction between $\mathrm{t}_{r e f}=\mathrm{t}\left(R_{\infty}\right)$ and $\mathrm{t}(R)$ ) when applied to the classical Crossland criterion and the new gradient criterion, respectively. With all such conceptions, the experimental data now agree very well with the ellipse arc based on the $f-t$ limits of the new criterion proposed (Eq. 57), as plotted in Fig. 8. It is also recalled [7] that the substitution of the material parameters by the bending and torsion limits is an unorthodox way to bypass the above described problems for classical criterion. The same ellipse formula is obtained in a more intrinsic way using the proposed criterion. The same approach can be applied to any other classical fatigue criterion. 


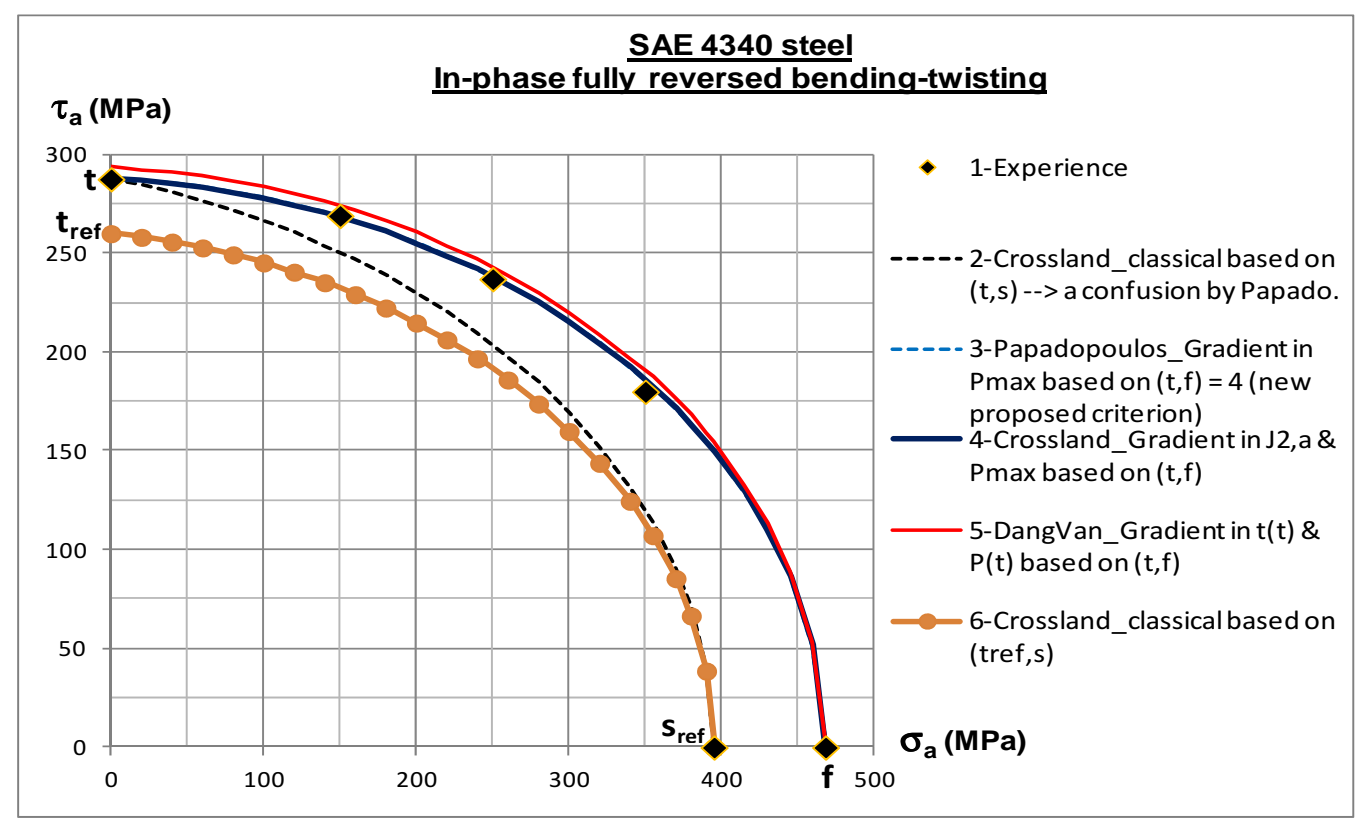

Fig. 8. Fully reversed combined bending-twisting fatigue limit data (Findley et al. [30], Papadopoulos and Panoskaltsis [7])

\section{Gradient Dang Van criterion}

A stress gradient dependent version of Dang Van criterion is proposed here in the same spirit as that of Crossland.

\subsection{Classical Dang Van criterion}

The Dang Van criterion presented in [32] is expressed as:

$$
\max _{t}\left\{\tau(t)+a_{D} P(t)\right\} \leq b_{D}
$$

$\tau(t)$ denotes the mesoscopic shear stress amplitude and is obtained from a mesoscopic stress tensor $\hat{\sigma}$ defined by:

$$
\hat{\boldsymbol{\sigma}}(t)=\left(\boldsymbol{\sigma}(t)-\boldsymbol{s}^{*}\right)
$$

$s^{*}$ is the center of the smallest hypersphere circumscribed to the loading path in deviatoric stress space. It is obtained by solving a "min-max" problem as follows:

$$
\boldsymbol{s}^{*}=\arg \min _{\boldsymbol{s}_{1}}\left\{\max _{t}\left\|\boldsymbol{s}(t)-\boldsymbol{s}_{1}\right\|\right\}
$$


In the case of fully reversed loading, the value $s^{*}=0$ can be directly deduced without solving the "min-max problem" as in general case.

Denoting by $\hat{\sigma}_{I I I}(t) \leq \hat{\sigma}_{I I}(t) \leq \hat{\sigma}_{I}(t)$ the principal stress values of stress tensor $\tilde{\sigma}$, one gets the amplitude of shear stress by:

$$
\tau(t)=\frac{1}{2}\left(\hat{\sigma}_{I}(t)-\hat{\sigma}_{I I I}(t)\right)
$$

$P(t)$ is the hydrostatic stress as a function of the time, given by:

$$
P(t)=\frac{\sigma_{k k}(t)}{3}
$$

The material characteristic parameters $a_{D}$ and $b_{D}$ of the Dang Van criterion, can be related to the fully reversed bending (or tension-compression because of the same stress state between them) fatigue limit, denoted by $f_{\text {ref }}$ (or $s_{\text {ref }}$ ), and to the torsion fatigue limit, denoted by $\mathrm{t}_{r e f}$,

$$
a_{D}=\frac{3 \mathrm{t}_{r e f}}{s_{r e f}}-3 / 2 ; \quad b_{D}=\mathrm{t}_{r e f}
$$

\subsection{Formulation of gradient Dang Van criterion}

Using as a basis the classical Dang Van criterion, Eq. (61), along with the general spirit, Eq. (9), for the development of a gradient version as below:

$$
\max _{t}\left\{\widetilde{\tau(t)}+a_{g} \widetilde{P(t)}\right\} \leq b_{g}
$$

The material parameters $a_{g}, b_{g}$ are actually equal to $a_{D}, b_{D}$ respectively, as was the case of the gradient Crossland criterion (sec. 4.2).

Using $\tau(t)$ as a basis, a new form $\widetilde{\tau(t)}$ embedded with gradient term is proposed:

$$
\widetilde{\tau(t)}=\tau(t)\left[1-\left(l_{\tau} \frac{\|\boldsymbol{Y}(t)\|}{\tau(t)}\right)^{n_{\tau}}\right]
$$

where $\boldsymbol{Y}(t)=\nabla \boldsymbol{\sigma}(t)$ and the definitions as well as significance of $n_{\tau}, l_{\tau}$ are the same as for the case of the Crossland (sec. 4.2). For $\widetilde{P(t)}$, the same form as that of 
[7] is proposed again:

$$
\widetilde{P(t)}=P(t)\left[1-\left\langle l_{\sigma} \frac{\|\boldsymbol{G}(t)\|}{P(t)}\right\rangle^{n_{\sigma}}\right]
$$

with the expressions of $\boldsymbol{G}(t),\|\boldsymbol{G}(t)\|$ similar to Eqs. $(29,30)$, the McCauley bracket $\langle 0\rangle$ similar to Eq. (31), and the definitions as well as significance of $n_{\sigma}, l_{\sigma}$ are the same.

The proposed criterion has six materials parameters $\left(a_{g}, b_{g}, l_{\tau}, l_{\sigma}, n_{\tau}, n_{\sigma}\right)$ to be identified experimentally.

\subsection{Calibration of the material parameters}

As previously, a procedure for obtaining the parameters from fully reversed torsion and fully reversed constant moment bending tests is detailed hereafter.

\section{a) Fully reversed torsion tests}

Applying first the gradient version described by Eq. (67) to the case of fully reversed torsion tests on specimen of radius $R$, with the fatigue limit denoted by $\mathrm{t}(R)$ and considering the most stressed points, relevant quantities are:

$$
\begin{aligned}
& \boldsymbol{\sigma}(t)=\sigma_{x \theta}(t)\left(e_{x} \otimes e_{\theta}+e_{\theta} \otimes e_{x}\right)=\mathrm{t}(R) \sin (\omega t)\left(e_{x} \otimes e_{\theta}+e_{\theta} \otimes e_{x}\right) \\
& \hat{\sigma}_{I}=\mathrm{t}(R)|\sin (\omega t)|, \quad \hat{\sigma}_{I I}=0, \quad \hat{\sigma}_{I I I}=-\mathrm{t}(R)|\sin (\omega t)| \\
& \tau(t)=\frac{1}{2}\left(\hat{\sigma}_{I}-\hat{\sigma}_{I I I}\right)=\mathrm{t}(R)|\sin (\omega t)| \\
& Y_{i j k} Y_{i j k}=2\left(\frac{\mathrm{t}(R) \sin (\omega t)}{R}\right)^{2} \text { and }\|Y(t)\|=\sqrt{2} \frac{\mathrm{t}(R)}{R}|\sin (\omega t)| \\
& \widetilde{\tau(t)}=\mathrm{t}(R)|\sin (\omega t)| L_{\tau}(R) \\
& \widetilde{P(t)}=0
\end{aligned}
$$

$$
\text { with: } \quad L_{\tau}(R)=1-2^{n_{\tau} / 2}\left(l_{\tau} / R\right)^{n_{\tau}}
$$

And using Eqs. (70, 71), the proposed fatigue criterion yields:

$$
\mathfrak{t}(R)=\frac{b_{D}}{L_{\tau}(R)} \approx \frac{\mathfrak{t}_{r e f}}{1-\left(l_{\tau} / R\right)^{n_{\tau}}}
$$

between two kinds of fatigue test can be clearly seen. As for the gradient Crossland criterion, this formula is used to calibrate the three material parameters $\tau_{\text {ref }}$, 
$l_{\tau}$ and $n_{\tau}$, using the experiment curve relating the fatigue limit $\mathrm{t}(R)$ to the radius of the specimen. The material parameters are then calibrated using the least square method on the tests points; and therefore the optimal parameters (i.e. the values which minimize the scatter between the predicted and experimental points) for the criterion are obtained. As an illustration, the torsion fatigue tests given by Massonnet [27] are used to identify $\tau_{r e f}, l_{\tau}$ and $n_{\tau}$, as shown in Fig. 6. A visual image of $\mathrm{t}_{r e f}$ aforementioned is as well found in this graph. The values obtained are: $\tau_{\text {ref }}=115$ $\mathrm{MPa}, l_{\tau}=9.810^{-1} \mathrm{~mm}$ and $n_{\tau}=0.5$.

\section{b) Fully reversed cantilever bending tests}

With the same notation and most stressed points to consider as in Sec. (4.3.c), all quantities are given by:

$$
\begin{aligned}
& \boldsymbol{\sigma}(t)=\sigma_{x x}(t) e_{x} \otimes e_{x}=f^{\prime}(R) \sin (\omega t) e_{x} \otimes e_{x} \\
& \|Y(t)\|=\frac{f^{\prime}(R)}{R}|\sin (\omega t)| \sqrt{1+\frac{R^{2}}{L^{2}}} \\
& \widetilde{\tau(t)}=\frac{f^{\prime}}{2}|\sin (\omega t)| L_{\tau f^{\prime}}(R, L)
\end{aligned}
$$

$$
\text { with: } \quad L_{\tau f^{\prime}}(R, L)=1-2^{n_{\tau}}\left(l_{\tau} / R\right)^{n_{\tau}}\left(1+\frac{R^{2}}{L^{2}}\right)^{n_{\tau} / 2}
$$

Similarly, using Eq. (69):

$$
\widetilde{P(t)}=\frac{f^{\prime}(R)}{3} \sin (\omega t) L_{\sigma f^{\prime}}(R, L)
$$

$$
\text { with: } \quad L_{\sigma f^{\prime}}(R, L)=1-\left(l_{\sigma} / R\right)^{n_{\sigma}}\left(1+\frac{R^{2}}{L^{2}}\right)^{n_{\tau} / 2}
$$

Finally, from Eq. (67), an equation with respect to the variable $f^{\prime}$ is solved to give:

$$
f^{\prime}(R)=\frac{s_{r e f}}{L_{\sigma f^{\prime}}-\frac{s_{r e f}}{2 \mathrm{t}_{r e f}}\left(L_{\sigma f^{\prime}}-L_{\tau f^{\prime}}\right)} \geq s_{r e f}
$$

The fatigue limit of four-point bending tests $f(R)$ can be directly obtained by imposing $L$ large enough such that $\frac{R^{2}}{L^{2}}$ in Eq. (78) is negligible and then $f^{\prime}(R) \equiv f(R)$. 


\section{Numerical implementation}

The stress gradient Crossland criterion, Eqs. (20), is considered as an illustration. The calculation of $\sqrt{\widetilde{J}_{2}}$, as described by Eq. (23), with the help of Eq. (16) or Eq. (15) for ${\sqrt{J_{2}}}_{a}$, and of Eq. (25) or (26) for $\|\boldsymbol{Y}(t)\|_{, a}$, is "min-max" or "max-max" problems in a 5-dimension space for $\sqrt{J_{2}, a}$ and 18-dimension space for $\|\boldsymbol{Y}\|_{, a}$.

Therefore, in numerical aspect, the calculation of $\sqrt{\widetilde{J}_{2, a}}$ is actually "min-max" or "max-max" problems with 23 variables. It is solved using user-written program under Matlab. It remains $\widetilde{P_{\max }}$ which evaluation through Eq. (28) is straightforward. Just using suitable operators in any available finite element code (i.e. Cast3M, [37]), the quantities $G=\nabla P_{\max }$ after Eq. (29) and then $\|G\|$ after Eq. (30) are estimated quickly.

So the proposed gradient fatigue criteria, Eqs. (20) and (67), can be numerically implemented within any available finite element code along with a user-written program to solve "min-max" or "max-max" problems.

\section{Discussion}

Remark 1 (Gradient terms) Limits of classical fatigue criteria in the literature are that the "size", gradient and loading effects are not captured. Even in the gradient fatigue criterion proposed by a number of authors such as the typical work of [7], the role of the shear stress gradient as well as the shear stress gradient amplitude in fatigue strength has not been made clear and thereby temporarily still neglected. In [7], the role of the shear stress gradient which is inherently assumed null only for some metals considered, but has been generally omitted even when applied to any other metal.

This study, as reasoned in the section 3.2, show that in some special cases where just one kind of load appears (e.g. pure torsion test, pure bending test), a unique gradient term is enough to model the gradient and loading effects. This is introduced either in the normal stress component of the classical fatigue criterion as [7] proposed, or in the shear stress part as presented in the current work. However, in multiaxial fatigue tests, concomitant two types of stress gradient terms are in principle indispensable to capture the previous effects. 
Remark 2 (Material characteristic length scale $\ell$ ) The values of $\ell$ of the model proposed extend from several hundredths of a millimeter to about a millimeter for cases considered, while the one of the model proposed very recently by Ferré et al. 2013 [22] takes about a micron. The very difference between them is physically explained by the following reason: we study here the fatigue endurance of macroscopic specimens and components for which the crack initiation is generally detected by loss of stiffness corresponding to crack length which can reach a millimeter; whereas Ferré et al. consider crack nucleation in the scale is few dozen microns.

Remark 3 (Insensitive threshold of effects) The dependence of fatigue limits on both "size" and gradient effects according to the specimen size (e.g. $L, R$ ) has a "saturated" or "insensitive" threshold. That means, there always exists a certain "saturated" value for the specimen size $\left(L_{\infty}, R_{\infty}\right)$ from which the fatigue behavior is insensitive to both effects and the proposed criteria exactly reduce to the respective classical ones.

Remark 4 (Approximation of some formulae) In the illustration through Crossland criterion, using a priori the exponent $n=0.5$ for some approximations (Eqs. (37), (53)) results in the very simple formulas for relevant quantities, especially in the combined bending-twisting case. This value of $n$ was afterwards affirmed reasonable through very good validation with some experimental classes. In the general case, however, $n$ could have another value for other experimental classes, then the proposed criteria may require to use the exact formulae, Eqs. $(75,77)$ e.g., to express consistently all relevant quantities (such as $L_{\tau f}, L_{\tau f^{\prime}}, L_{\sigma f}, L_{\sigma f^{\prime}} \ldots$ ) in any case of test according to their analogues in calibration tests (torsion and bending tests).

\section{Conclusion}

The present study develops a simple formulation of gradient multiaxial fatigue criteria extending the classical HCF criteria. The objective is to model the "size", surface gradient and loading effects, not included yet in classical mechanics but 
become important at small scale, by taking into account just the gradient effect.

Basing on some experimental observations, and departing from classical fatigue criteria, new class of criteria with stress gradient terms entering not only in the normal stress but also in the shear stress amplitude, are proposed. Such a formulation allows the new criteria to capture the "size" and gradient effects, and to cover a large range of loading mode (traction, bending, shearing). These new criteria are then generalized to multiaxial cases to capture both well-known phenomena "Smaller is Stronger" and "Higher Gradient is Stronger" and thus can reproduce fatigue experimental data even at small scale. Here in this work, the nature of these two phenomena is also clarified. "Higher Gradient is Stronger" is only related to the gradient effect, while "Smaller is Stronger" is related to both pure size and gradient effects where the latter is dominant - rather than totally to the pure size effect as usually believed.

Extensions of some classical fatigue limit criteria such Crossland and Dang Van are done as illustrations. The proposed criteria shown a good agreement with a number of experiments from the literature. A more comprehensive validation for complex loading (real multiaxial loads) could be perspective for this research direction.

Nevertheless, in this work only cases with critical points located at the specimens surfaces have been examined. In these cases, the gradient is such that it has a beneficial effect on fatigue. However, cases where the effect can be presumably negative, especially with the presence of residual stresses, can be encountered. A reexamination of the approach will be the object of the further work. Besides, for notched fatigue problems, this approach may be still applicable.

In conclusion, the extension of classical fatigue criteria embedding into them two gradient terms, one corresponds to normal stress part, the other to shear stress part, leads to new versions able to describe common effects on multiaxial fatigue endurance.

\section{References}

[1] Amargier R., Fouvry S., Chambon L., Schwob C., Poupon C. Stress gradient effect on crack initiation in fretting using a multiaxial fatigue framework, Int J Fatigue 2010; 32(12):1904-12. 
[2] Taylor D. The theory of critical distances: a new perspective in fracture mechanics. Oxford, UK: Elsevier; 2007.

[3] Araujo JA, Susmel L, Taylor D, T Ferro JC, Mamiya EN. On the use of the theory of critical distances and the Modified Wohler curve method to estimate fretting fatigue strength of cylindrical contacts. Int J Fatigue 2007; 29:95-107.

[4] Pluvinage G. Notch effect in high cycle fatigue. ICF 9 Proceedings, 1997; 3:1237-50.

[5] Qylafku G., Azari Z., Kadi N., Gjonaj M. , Pluvinage G. Application of a new model proposal for fatigue life prediction on notches and key-seats, Int J Fatigue 1999; 21(8):753-60.

[6] Maitournam, M. H., Van, K. D. and Flavenot, J.-F. Fatigue Design of Notched Components with Stress Gradients and Cyclic Plasticity. Adv. Eng. Mater. 2009; $11: 750-754$.

[7] Papadopoulos I.V., Panoskaltsis V.P. Invariant formulation of a gradient dependent multiaxial high-cycle fatigue criterion. Engineering Fracture Mechanics 1996; 55-No. 4:513-528.

[8] Palin-Luc T. Stress gradient and size effects in multiaxial fatigue. In Materials Week 2000 (Munich, Germany).

[9] Banvillet A., Palin-Luc T., Lasserre S. A volumetric energy based high cycle multiaxial fatigue citerion. Int J of Fatigue 2003; 25-No. 8:755-769.

[10] Palin-Luc T. Approche énergétique et volumique de la fatigue multiaxiale des mtaux. Habilitation diriger des recherches (HDR) 2004.

[11] Morel F., Palin-Luc T. A non-local theory devoted to high cycle multiaxial fatigue. Int J Fatigue 2002; 25:649-65.

[12] Weber B. Fatigue multiaxiale des structures industrielles sous chargement quelconque. PhD thesis 1999, INSA de Lyon.

[13] Phillips C.E., Heywood R.B. The size effect in fatigue of plain and notched steel specimens loaded under reversed direct stress. I. Mech. E. 1951; 165:113-124.

[14] Pogoretskii R.G., Karpenko G.V. Effect of test piece length on the fatigue strength of steel in air. Fiziko Khimicheskaya Mekhanika Materialov 1965; 1:90-94. 
[15] Maitournam MH, Pommier B, Thomas JJ. Determination of the asymptotic response of a structure under cyclic thermomechanical loading. Comp Rend Mec 2002; 330(10):703-8.

[16] Flavenot J-F., Skally N. L'épaisseur de couche critique ou une nouvelle approche du calcul en fatigue a long terme des structures soumises a des solicitations multiaxialles en présence de gradient de contraintes. Rev. Méca. Matériaux et Electricité 1983; 397:1525.

[17] Sonsino C.M., Kaufmann H., Grubisic V. Transferability of material data for the example of a randomly loaded forged truck stub axle. SAE Technical Paper - J of Materials and Manufacturing 1997; No. 970708:1-22.

[18] Palin-Luc T., Lasserre S. An energy based criterion for high cycle multiaxial fatigue. European Journal of Mechanics - A/Solids 1998; 17(2):237-251.

[19] Brand A., Sutterlin R. Calcul des piéces a la fatigue. Méthode du gradient. In Senlis: Editions du CETIM 1980; 157p.

[20] Brand A. Nouvelle méthode de calcul en fatigue faisant intervenir le gradient de contraintes. Mécanique Matériaux Electricité 1981; N0 375-376-377:136-143.

[21] Ngargueudedjim K., Weber B., Robert J-L. Accounting for stress gradient effect in multiaxial fatigue criteria. de Freitas M, editor. Sixth Int Conf on Biaxial/Multiaxial Fatigue and Fracture. ESIS 2001; 143-50.

[22] Ferre R., Fouvry S., Berthel B., Ruiz-sabariego J.-A. Stress gradient effect on the crack nucleation process of a Ti-6Al-4V titanium alloy under fretting loading: comparison between non-local fatigue approaches. Int. J. of Fatigue, 2013, Accepted Manuscrit.

[23] Robert J-L. Contribution a l'étude de la fatigue multiaxiale sous sollicitations périodiques ou aléatoires. These de l'Institut National des Sciences Appliquées (INSA) de Lyon 1992; $N^{0} 92$ ISAL 0004:229p.

[24] Fogue M., Bahuaud J. Fatigue multiaxiale a durée de vie illimitée. $7^{\text {eme }}$ Congres Français de Mécanique 1985, Bordeaux, pp.30-31.

[25] Fogue M. Critere de fatigue a longue durée de vie pour des états multiaxiaux de contraintes sinusoidales en phase et hors phase. These de l'Institut National des Sciences Appliquées (INSA) de Lyon 1987; $N^{0} 87$ ISAL 0030:189p. 
[26] Pavan A. Contribution aux calculs d'organes d'ensembles mecaniques par rapport a la limite de fatigue. Explication des principaux facteurs. PhD thesis 1979, Universit de Reims, Reims, France.

[27] Massonnet Ch. Le dimensionnement des pices de machines soumises la fatigue. Contribution exprimentale d'etude de l'effet de l'echelle et des entailles. Revue Universelle des Mines 1955; 9:203-222.

[28] Massonnet Ch. The effect of size, shape and grain size on the fatigue strength of medium carbon steel. In ASTM (American Society for Testing and Materials Int) 1956; 56:954-978.

[29] Crossland B. Effect of large hydrostatic pressures on the torsional fatigue strength of an alloy steel. Int Conf on Fatigue of Metals, London, U.K, I. Mech. E. 1956; 138-149.

[30] Findley W.N., Coleman J.J., Hanley B.C. Theory for combined bending and torsion fatigue with data for SAE 4340 steel. Int Conf on Fatigue of Metals, London, England, I. Mech. E. 1956; 150-157.

[31] Papadopoulos I., Davoli P., Gorla C., Filippini M., Bernasconi A. A comparative study of multiaxial high-cycle fatigue criteria for metals. Int J Fatigue 1997;19(3):219-35.

[32] Ballard P., Van K.D., Deperrois A., Papadopoulos Y.V. High cycle fatigue and a finite element analysis. Fatigue \& Fracture of Engineering Materials \& Structures, 1995, 18 : 397-411.

[33] Moore H.F., Morkovin D. Third progress report on the effect of size of specimen on fatigue strength of three types of steel. ASTM (American Society for Testing and Materials Int) 1944; No. 44:137-158.

[34] Nguyen Q.S. Variational principles in the theory of gradient plasticity. Comptes Rendus Mecanique 2011; No.12:339:743-750.

[35] Palin-Luc T. Fatigue multiaxiale d'une fonte GS sous sollicitations combines d'amplitude variable. PhD thesis, ENSAM, Talence, 1996.

[36] Sines G. Behavior of metals under complex static and alternating stresses. Metal Fatigue 1959; 145-169.

[37] CEA, DEN, DM2S, SEMT, Cast3M, <http://www-cast3m.cea.fr/>. 


\section{List of figures}

- Fig. 1: Stress distribution types in fatigue tests of the same specimen: (a) tensioncompression vs. bending tests; (b-c-d) tension-compression vs. rotative bending vs. plane bending (cf. Weber [12])

- Fig. 1: Comparison between the fully reversed tension-compression and rotative bending fatigue limits of smooth specimens with the same geometry and material, for different materials (Results of Palin-Luc [35], synthesized by Weber [12])

- Fig. 2: Evolution of the fully reversed tension-compression and rotative bending fatigue limits of smooth specimens with the same geometry and material according to their radii (Results of Massonnet [27], synthesized by Weber [12])

- Fig. 3: Four-point bending (constant moment) and cantilever bending tests: (a) four-point bending; (b) cantilever bending [7].

- Fig. 4: Constant moment bending fatigue limit data: (a) constant radius $R$; (b) constant length L (Results of Pogoretskii and Karpenko [14], represented by Weber [12])

- Fig. 6: Fully reversed torsion fatigue limit of smooth cylindrical samples (cf. Massonnet [28])

- Fig. 7: Fully reversed bending fatigue limits of cylindrical specimen (Massonnet [27], Moore \& Morkovin [33], Pogoretskii \& Karpenko [14], Papadopoulos \& Panoskaltsis [7])

- Fig. 8: Fully reversed combined bending-twisting fatigue limit data (Findley et al. [30], Papadopoulos and Panoskaltsis [7])

\section{List of tables}

Tab 1: Comparison between the fully reversed tension-compression and rotative bending fatigue limits of smooth specimens with the same geometry and material, for different materials (Results of Palin-Luc [35], synthesized by Weber [12])

Tab 2: Comparison between the fully reversed rotative bending and plane bending fatigue limits for different metals of smooth specimens of the same geometry and material (Results of Palin-Luc [35], synthesized by Weber [12]) 


\section{Highlights}

- Formulation of gradient fatigue criteria to capture the gradient and loading effects

- Gradients of the shear stress amplitude and the hydrostatic stress are simultaneously introduced

- Gradient versions of Crossland and Dang Van criteria are provided

- Experimental observations of the gradient and loading effects are reproduced 